\title{
Synthesis, Fungitoxic Activity against Botrytis cinerea and Phytotoxicity of Alkoxyclovanols and Alkoxyisocaryolanols
}

\author{
Adriana de Almeida Pinto Bracarense ${ }^{1,2}$, Jociani Ascari ${ }^{1,3}{ }^{10}$, Giovanni Gontijo de Souza ${ }^{1}$, Thays Silva Oliveira ${ }^{1}$, \\ Antonio Ruano-González ${ }^{4}{ }^{(0)}$, Ana A. Pinto ${ }^{4}$, Maria Amélia Diamantino Boaventura ${ }^{1}$,

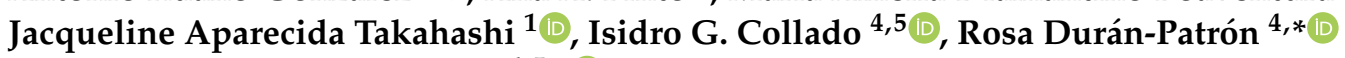 \\ and Antonio J. Macías-Sánchez ${ }^{4,5, *(\mathbb{D})}$
}

check for updates

\section{Citation: de Almeida Pinto}

Bracarense, A.; Ascari, J.; de Souza,

G.G.; Oliveira, T.S.; Ruano-González, A.; Pinto, A.A.; Boaventura, M.A.D.; Takahashi, J.A.; Collado, I.G.; Durán-Patrón, R.; et al. Synthesis, Fungitoxic Activity against Botrytis cinerea and Phytotoxicity of Alkoxyclovanols and Alkoxyisocaryolanols. J. Fungi 2021, 7, 1079. https://doi.org/10.3390/ jof7121079

Academic Editor: Milena Cotorás

Received: 22 November 2021 Accepted: 10 December 2021 Published: 15 December 2021

Publisher's Note: MDPI stays neutral with regard to jurisdictional claims in published maps and institutional affiliations.

Copyright: (c) 2021 by the authors. Licensee MDPI, Basel, Switzerland. This article is an open access article distributed under the terms and conditions of the Creative Commons Attribution (CC BY) license (https:/ / creativecommons.org/licenses/by/ $4.0 /)$.
1 Departamento de Química, Instituto de Ciências Exatas, Universidade Federal de Minas Gerais, Av. Presidente Antônio Carlos, 6627, Belo Horizonte 31270-901, MG, Brazil; adrianabracarense@gmail.com (A.d.A.P.B.); jascari@utfpr.edu.br (J.A.); giovannigs1@yahoo.com.br (G.G.d.S.); tsoliveira3@yahoo.com.br (T.S.O.); dianadb@netuno.lcc.ufmg.br (M.A.D.B.); jat@qui.ufmg.br (J.A.T.)

2 Departamento de Química, Centro Federal de Educação Tecnológica de Minas Gerais, Av. Amazonas, 5253, Belo Horizonte 30421-169, MG, Brazil

3 Coordenaçao Ciências Biológicas, Universidade Tecnológica Federal do Paraná, Prolongamento da Rua Cerejeira, s/n, Santa Helena 85892-000, PR, Brazil

4 Departamento de Química Orgánica, Facultad de Ciencias, Universidad de Cádiz, Puerto Real, 11510 Cádiz, Spain; antonio.ruano@uca.es (A.R.-G.); ana.pinto@mail.uca.es (A.A.P.); isidro.gonzalez@uca.es (I.G.C.)

5 Instituto de Investigación en Biomoléculas (INBIO), Universidad de Cádiz, Puerto Real, 11510 Cádiz, Spain

* Correspondence: rosa.duran@uca.es (R.D.-P.); antoniojose.macias@uca.es (A.J.M.-S.); Tel.: +35-956-012704 (A.J.M.-S.)

Abstract: Clovane and isocaryolane derivatives have been proven to show several levels of activity against the phytopathogenic fungus Botrytis cinerea. Both classes of sesquiterpenes are reminiscent of biosynthetic intermediates of botrydial, a virulence factor of $B$. cinerea. Further development of both classes of antifungal agent requires exploration of the structure-activity relationships for the antifungal effects on $B$. cinerea and phytotoxic effects on a model crop. In this paper, we report on the preparation of a series of alkoxy-clovane and -isocaryolane derivatives, some of them described here for the first time $(\mathbf{2 b}, \mathbf{2 d}, \mathbf{2 f}-\mathbf{2 h}$, and $\mathbf{4 c - 4 e )}$; the evaluation of their antifungal properties against $B$. cinerea, and their phytotoxic activites on the germination of seeds and the growth of radicles and shoots of Lactuca sativa (lettuce). Both classes of compound show a correlation of antifungal activity with the nature of side chains, with the best activity against $B$. cinerea for $\mathbf{2 d}, \mathbf{2} \mathbf{h}, \mathbf{4} \mathbf{c}$ and $\mathbf{4 d}$. In general terms, while 2-alkoxyclovan-9-ols (2a-2e) exert a general phytotoxic effect, this is not the case for 2-arylalkoxyclovan-9-ols (2f-2i) and 8-alkoxyisocaryolan-9-ols (4a-4d), where stimulating effects would make them suitable candidates for application to plants.

Keywords: Botrytis cinerea; antifungal; phytotoxic activity; clovane; isocaryolane; Lactuca sativa

\section{Introduction}

Fungi of the Botrytis (Sclerotiniaceae) genus are involved in substantial crop losses in economically relevant cultivars. While most species are host specific, $B$. cinerea has a wide host range which involves hundreds of vascular plant genera [1]. Infection is usually promoted by high humidity conditions, involving a variety of pathways to achieve infection and colonization [2]. Chemical control based on synthetic fungicides [3] is still the prevalent protection option for many crops, but, their increasing impact on the environment [4], concerns on residue levels and effective delivery [5], and the ever-present acquired resistance phenomenon [6], are forcing a rethinking of the way strategies of chemical control are used [7]. 
On the other hand, in the context of integrated pest management [8], there is an increasing interest in the use of biopesticides [9], which comprises the use of living microorganisms and substances of natural origin, such as plant and microbial extracts, as well as natural products isolated from the abovementioned sources or closely related derivatives (selected examples can be found from plants [10-12], microorganisms [13-16] and endophytes [17-19]). Therefore, there is a need for the development of novel chemical control agents, capable of being used in conjunction with living microorganisms and with the ability to antagonize $B$. cinerea, which calls for aiming at selective targets in the fungal infection cycle. Evidence of the protective role of endophytic microorganisms against the attack of $B$. cinerea on relevant cultivars $[20,21]$ stresses the need for chemical control agents with selectivity against the phytopathogen, as this would reduce the impact on the plant microbiome.

It has been reported that commercial fungicides have an impact on plant physiology [22]. For instance, the treatment of Vitis vinifera with fludioxonil and pyrimethanil, used in the control of $B$. cinerea, impairs photosynthesis $[23,24]$, while mancozeb, an extensively used contact fungicide, induces oxidative damage on L. sativa [25]. Therefore, the development of bioinspired antifungal agents should be undertaken keeping in mind these undesired effects.

Botrydial is a low molecular weight phytotoxin [26], with a carbon skeleton not following the isoprene rule, which originates from farnesyl diphosphate by folding, cyclization, and a sequence of oxidative transformations [27-30]. Genes encoding for the relevant steps of the metabolic pathway to botrydial and related compounds have also been elucidated [31,32]. This secondary metabolite is part of the interaction of the fungus with the host plant, inducing cell death [33]. The modulation of its biosynthesis may lead to the control of the fungus through a selective mechanism, as symptoms of the disease can be decreased by inhibition of the production of the toxin in deleted mutants [34].

Our research group has approached the control of the fungus $B$. cinerea by developing nonphytotoxic analogs of the biosynthetic intermediates of the phytotoxin botrydial. A variety of compounds with different sesquiterpenic carbon skeletons have been prepared for this purpose [26].

Clovane and isocaryolane skeleton compounds are caryophyllene cyclization derivatives [35,36], which are reminiscent of botrydial biosynthetic intermediates such as 1(Figure 1). Within this context, 2-alkoxyclovan-9-ols, obtained by the cyclization of caryophyllene oxide in alcohols, catalyzed by TCNE [37], have shown different levels of activity, which are correlated with the nature of the substituent at C-2 [38]. On the other hand, the metabolism of selected 2-alkoxyclovan-9-ols has shown the preference of the fungus for the dealkylation of the side chain at C-2, yielding less active compounds $[39,40]$.

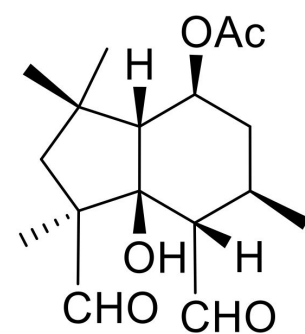

Botrydial

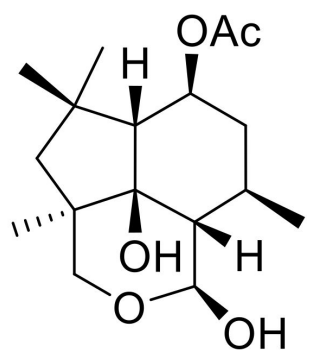

Dihydrobotrydial

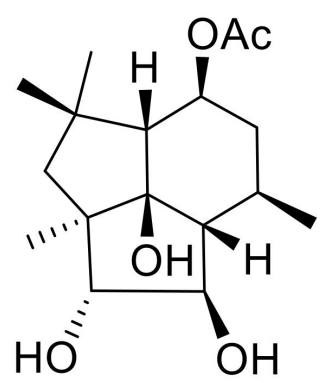

1

Figure 1. Metabolites from Botrytis cinerea.

Reported antifungal activities of clovane derivatives show a general decrease of the activity when further hydroxyl groups are introduced, or when the side chain at C-2 is absent $[39,40]$. On the other hand, QSAR calculations suggest an increase in the fungal activity if the lipophilicity of the side chain at C-2 is increased [38]. Evaluation of the 
antifungal activity of a homologous series of 2-alkoxyclovan-9-ol derivatives and some systematic variations of active 2-(aryl)alkoxyclovan-9-ols described before [38], should shed some light on the extent and limitations of this lipophylic effect.

8-Methoxyisocaryolan-9-ol, an isocaryolane derivative, has also shown an interesting level of antifungal activity against the phytopathogen B. cinerea [41]. Incubation of this compound and related substrates like isocaryolane-8,9-diol and isocaryolan-9-ol showed, on one hand, that these compounds interfered with the production of botryane metabolites, like dihydrobotrydial (Figure 1), a metabolic sink of botryane metabolism [42]. On the other hand, it was apparent that the presence of a methoxy group at C-8 was a key feature for the antifungal activity of isocaryolane derivatives; this ether was cleaved by fungal metabolism and antifungal activity decreased as the hydroxylation level increased [41,43]. Therefore, evaluation of a homologous series of 8-alkoxyisocaryolan-9-ol derivatives should shed some light on the suitability of isocaryolane compounds as antifungal leads.

Another aspect to consider in the development of clovane or isocaryolane derivatives as effective fungal leads, is the extent of the phytotoxic effect exerted by these compounds, which should be reduced as much as possible if the compounds of interest are to find practical applications in the field.

Therefore, in this paper, we report the preparation of a homologous series of 2alkoxyclovan-9-ols (2a-2e), some related to 2-arylethoxyclovan-9-ols (2f-2i), as well as a homologous series of 8-alkoxyisocaryolan-9-ols (4a-4e). We also evaluate their antifungal properties against $B$. cinerea, and their phytotoxic activities on the germination of seeds and growth of radicles and shoots of L. sativa (lettuce) [44-47], a plant successfully used in the evaluation of the phytotoxicity of bioactive natural products.

\section{Materials and Methods}

\subsection{General Experimental Procedure}

Melting points were measured with a Reichert-Jung Kofler block and are uncorrected. Optical rotations were determined on a Perkin-Elmer 341 polarimeter. IR spectra were recorded on a Perkin-Elmer Spectrum BX FT-IR spectrophotometer. ${ }^{1} \mathrm{H}$ and ${ }^{13} \mathrm{C}$ NMR spectra were obtained on a Varian INOVA 400 NMR spectrometer using tetramethylsilane as an internal reference. NMR assignments were made by a combination of $1 \mathrm{D}$ and 2D techniques and by comparison with assignments available in the literature for previously described compounds, where appropriate. Mass spectra were recorded on a Finnigan Voyager spectrometer at $70 \mathrm{eV}$. High resolution mass spectra were recorded on a Micromass Autospec spectrometer at $70 \mathrm{eV}$ or on a Waters Synapt G2 QTOF spectrometer in ESI mode. HPLC was performed with a Hitachi/Merck L-6270 apparatus equipped with a UV-VIS detector (L 4250) and a differential refractometer detector (RI-71). TLC was performed on Merck Kiesegel 60 F254 plates, with a $0.2 \mathrm{~mm}$ thick film. Silica gel (Merck) was used for column chromatography. Purification by HPLC was performed using a Si gel column (LiChrospher Si 60, $10 \mu \mathrm{m}, 1 \mathrm{~cm}$ wide, $25 \mathrm{~cm}$ long).

\subsection{General Procedure for the Alcoholysis of Caryophyllene Oxide Catalyzed by TCNE}

(-)-Caryophyllene oxide, dissolved in alcohol, was treated with a catalytic amount of TCNE at room temperature (see Table 1 for details). When the epoxide was consumed, as shown by TLC monitoring, the solvent was either evaporated under vacuum or washed with brine. The resulting gum was dissolved in ethyl acetate and dried over anhydrous $\mathrm{Na}_{2} \mathrm{SO}_{4}$. After filtration, evaporation under reduced pressure of the solvent afforded a crude reaction product. Purification by column chromatography on silica gel, with increasing gradients of ethyl acetate in petroleum ether, combined with HPLC purification, afforded 2a-2g (Figure 2). 
Table 1. Alcoholysis of Caryophyllene Oxide Catalyzed by TCNE. Reaction Conditions, Products and Yields.

\begin{tabular}{ccccc}
\hline Epoxide (mg) & Alcohol (mL) & TCNE (mg) & Time (hr) & Product (Yield) \\
\hline 1019 & $\mathrm{CH}_{3} \mathrm{CH}_{2} \mathrm{OH}(20)$ & 63 & 36 & $2 \mathrm{~b}(15 \%)$ \\
1013 & $\mathrm{CH}_{3}\left(\mathrm{CH}_{2}\right)_{3} \mathrm{OH}(20)$ & 61 & 36 & $2 \mathrm{~d}(11 \%)$ \\
500 & $\mathrm{PhCH}_{2} \mathrm{CH}_{2} \mathrm{OH}(3)$ & 58 & 103 & $2 \mathrm{f}(10 \%)$ \\
500 & $\mathrm{PhO}\left(\mathrm{CH}_{2}\right)_{2} \mathrm{OH}(0.5)$ & 60 & 114 & $2 \mathrm{~g} \mathrm{(9 \% )}$ \\
\hline
\end{tabular}

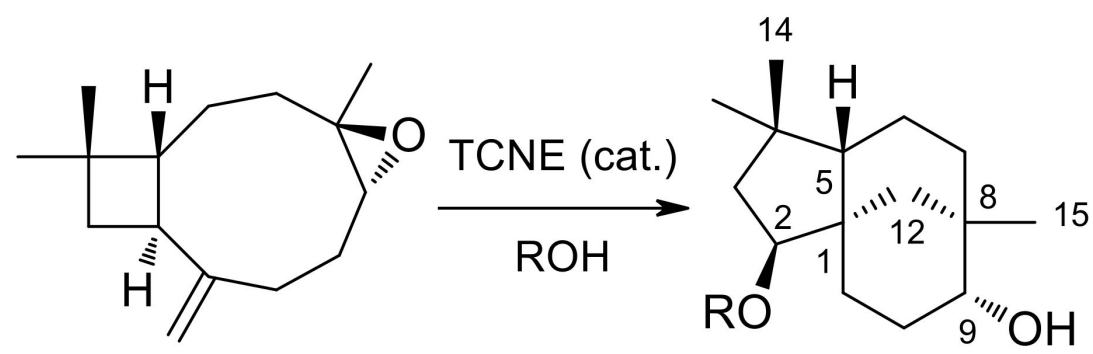

\section{Caryophyllene oxide}

$$
\begin{gathered}
\mathrm{R}=\left(\mathrm{CH}_{2}\right)_{n} \mathrm{CH}_{3} \\
\mathrm{n}=0-4 \\
\mathrm{R}=\left(\mathrm{CH}_{2}\right)_{2} \mathrm{Ph} \\
\mathrm{R}=\left(\mathrm{CH}_{2}\right)_{2} \mathrm{OPh}
\end{gathered}
$$

2a $\mathrm{R}=\mathrm{CH}_{3}$

2b R $=\left(\mathrm{CH}_{2}\right) \mathrm{CH}_{3}$

2c $\mathrm{R}=\left(\mathrm{CH}_{2}\right)_{2} \mathrm{CH}_{3}$

2d R $=\left(\mathrm{CH}_{2}\right)_{3} \mathrm{CH}_{3}$

2e $\mathrm{R}=\left(\mathrm{CH}_{2}\right)_{4} \mathrm{CH}_{3}$

2f $\mathrm{R}=\left(\mathrm{CH}_{2}\right)_{2} \mathrm{Ph}$

2g R $=\left(\mathrm{CH}_{2}\right)_{2} \mathrm{OPh}$

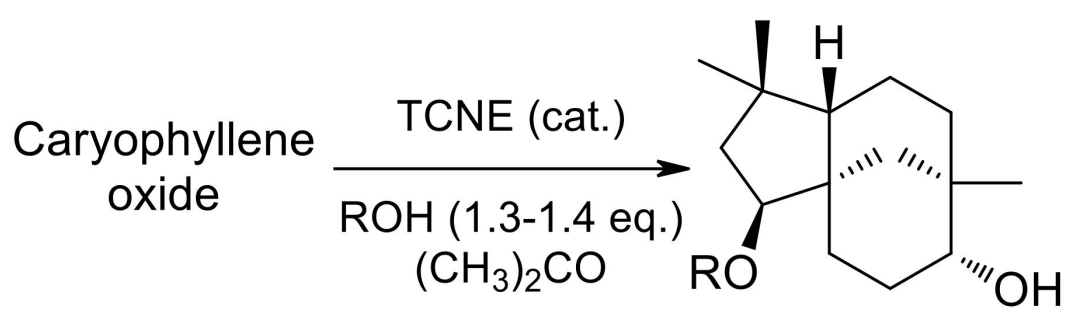

$$
\begin{aligned}
& \mathrm{R}=\left(\mathrm{CH}_{2}\right)_{2} \mathrm{Ph}\left(p-\mathrm{NO}_{2}\right) \\
& \mathrm{R}=\left(\mathrm{CH}_{2}\right)_{2} \mathrm{OPh}\left(p-\mathrm{NO}_{2}\right)
\end{aligned}
$$

2h R $=\left(\mathrm{CH}_{2}\right)_{2} \mathrm{Ph}\left(p-\mathrm{NO}_{2}\right)$

2i $\mathrm{R}=\left(\mathrm{CH}_{2}\right)_{2} \mathrm{OPh}\left(p-\mathrm{NO}_{2}\right)$

Figure 2. Synthesis of 2-Alkoxyclovan-9-ols (2a-2e) and 2-Arylethoxyclovan-9-ols (2f-2i).

$(2 S, 9 R)$-2-Methoxyclovan-9-ol (2a), (2S,9R)-2-propoxyclovan-9-ol (2c), and (2S,9R)2-pentoxyclovan-9-ol (2e) were obtained according to procedures described in the literature $[37,48]$. Reaction conditions, products and yields for $\mathbf{2} \mathbf{b}, \mathbf{2} \mathbf{d}$, and $\mathbf{2} \mathbf{f}, \mathbf{2} \mathbf{g}$ can be found in Table 1.

\subsubsection{Spectroscopic Data of Compounds $\mathbf{2} \mathbf{b}, \mathbf{2} \mathbf{d}, \mathbf{2 f}, \mathbf{2} \mathbf{g}$}

(2S,9R)-2-ethoxyclovan-9-ol (2b): needles $\left(\mathrm{CHCl}_{3}\right) ; 58-60{ }^{\circ} \mathrm{C} ;[\alpha]_{D}^{25}+1.5\left(\right.$ c 3.8, $\left.\mathrm{CDCl}_{3}\right)$; IR (film) $v_{\max } 3422,2927,2864,1459,1365,1107 \mathrm{~cm}^{-1} ;{ }^{1} \mathrm{H}$ NMR data $\left(\mathrm{CDCl}_{3}, 400 \mathrm{MHz}\right)$, see Table 2; ${ }^{13} \mathrm{C}$ NMR data $\left(\mathrm{CDCl}_{3}, 100 \mathrm{MHz}\right.$ ), see Table 3; HMBC (selected correlations) C- $1 \rightarrow \mathrm{H}-3 \alpha, \mathrm{H}-3 \beta, \mathrm{H}-5 \beta, \mathrm{H}-6 \mathrm{~b}, \mathrm{H}-10 \alpha, \mathrm{H}-11 \mathrm{a}, \mathrm{H}-12 \mathrm{a}$; C-2 $\rightarrow \mathrm{H}-3 \alpha, \mathrm{H}-3 \beta, \mathrm{H}-12 \mathrm{a}$, H-1'a,b; C- $4 \rightarrow$ H-3 $\alpha$, H-3 $\beta$, H-5 $\beta$, H-13 $\alpha$, H-14 $\beta$; C- $8 \rightarrow$ H-5 $\beta$, H-7a, H-7b, H-10 $\alpha$, H-12a; $\mathrm{C}-15 \rightarrow$ H-7a, H-12a; C-1 ${ }^{\prime} \rightarrow$ H-2 $\alpha$, H-2 $\beta ;$ C-2 ${ }^{\prime} \rightarrow$ H-1'a,b; EIMS $m / z 266\left[\right.$ [M] $^{+}$(19), 251 (16), 
220 (13), 205 (20), 192 (21), 161 (20), 151 (12), 135 (26), 113 (100), 95 (16), 85 (33); HREIMS $\mathrm{m} / \mathrm{z} 266.2268[\mathrm{M}]^{+}$(calcd for $\mathrm{C}_{17} \mathrm{H}_{30} \mathrm{O}_{2}, 266.2246$ ).

Table 2. ${ }^{1} \mathrm{H}$ NMR Spectroscopic Data $\left(\mathrm{CDCl}_{3}, 400 \mathrm{MHz}\right)$ for Compounds $\mathbf{2} \mathbf{b}, \mathbf{2} \mathbf{d}$, and $\mathbf{2} \mathbf{f}-\mathbf{2 h}$.

\begin{tabular}{|c|c|c|c|c|c|}
\hline & $2 b$ & $2 d$ & $2 f$ & $2 g$ & $2 \mathrm{~h}$ \\
\hline Position & $\delta_{\mathrm{H}}, \operatorname{Mult}(J$ in $\mathrm{Hz})$ & $\delta_{\mathrm{H}}$, Mult $(J$ in $\mathrm{Hz})$ & $\delta_{\mathrm{H}}$, Mult $(J$ in $\mathrm{Hz})$ & $\delta_{\mathrm{H}}$, Mult $(J$ in $\mathrm{Hz})$ & $\delta_{\mathrm{H}}, \operatorname{Mult}(J$ in $\mathrm{Hz})$ \\
\hline $2 \alpha$ & $3.40, \mathrm{dd}(10.6,5.6)$ & 3.36 , dd $(10.3,5.6)$ & $3.32, \mathrm{dd}(10.2,5.7)$ & $3.44, \mathrm{dd}(10.3,5.6)$ & $3.34, \mathrm{dd}(9.8,5.6)$ \\
\hline $3 \alpha$ & $1.66, \mathrm{dd}(11.8,5.6)$ & $1.65, \mathrm{dd}(11.9,5.5)$ & $1.58, \mathrm{dd}(11.9,5.7)$ & $1.64, \mathrm{dd}(12.0,5.6)$ & $1.61, \mathrm{dd}(11.8,5.6)$ \\
\hline $3 \beta$ & 1.48 , dd $(11.8,10.6)$ & $1.48, \mathrm{~m}$ & $1.40, \mathrm{dd}(11.9,10.3)$ & $1.47, \mathrm{dd}(12.0,10.3)$ & $1.41, \mathrm{dd}(11.8,9.8)$ \\
\hline $5 \beta$ & $1.38, \mathrm{~m}$ & $1.38, \mathrm{~m}$ & $1.31, \mathrm{~m}$ & $1.34, \mathrm{~m}$ & $1.38, \mathrm{~m}$ \\
\hline $6 a$ & $1.38, \mathrm{~m}$ & $1.40, \mathrm{~m}$ & $1.35-1.27, \mathrm{~m}$ & $1.33, \mathrm{~m}$ & $1.40-1.20, \mathrm{~m}$ \\
\hline $6 \mathrm{~b}$ & $1.31, \mathrm{~m}$ & $1.30, \mathrm{~m}$ & $1.35-1.27, \mathrm{~m}$ & $1.23, \mathrm{~m}$ & $1.40-1.20, \mathrm{~m}$ \\
\hline $7 \mathrm{a}$ & $1.38, \mathrm{~m}$ & $1.36, \mathrm{~m}$ & $1.29, \mathrm{~m}$ & $1.30, \mathrm{~m}$ & $1.35, \mathrm{~m}$ \\
\hline $7 \mathrm{~b}$ & $1.09, \mathrm{~m}$ & $1.10, \mathrm{~m}$ & $1.02, \mathrm{~m}$ & $1.03, \mathrm{~m}$ & $1.08, \mathrm{~m}$ \\
\hline $9 \beta$ & 3.30 , brs & $3.29, \mathrm{brs}$ & 3.21 , brs & 3.23, brs & 3.29 , brs \\
\hline $10 \alpha$ & $1.58, \mathrm{~m}$ & $1.59, \mathrm{~m}$ & $1.50, \mathrm{~m}$ & $1.51, \mathrm{~m}$ & $1.56, \mathrm{~m}$ \\
\hline $10 \beta$ & $1.96, \operatorname{tdd}(14.2,4.8,3.3)$ & $1.96, \operatorname{tdd}(14.2,5.0,3.2)$ & $1.89, \operatorname{tdd}(14.2,5.9,3.5)$ & 1.90, tdd $(14.3,5.0,3.3)$ & $1.93, \operatorname{tdd}(14.1,6.8,4.5)$ \\
\hline $11 \mathrm{a}$ & $1.69, \mathrm{dd}(13.7,4.8)$ & $1.69, \mathrm{~m}$ & $1.56, \mathrm{~m}$ & $1.63, \mathrm{~m}$ & $1.59, \mathrm{~m}$ \\
\hline $11 b$ & $1.09, \mathrm{~m}$ & $1.11, \mathrm{~m}$ & $1.00, \mathrm{~m}$ & $1.06, \mathrm{~m}$ & $1.09, \mathrm{~m}$ \\
\hline $12 \mathrm{a}$ & $1.59, \mathrm{~d}(12.7)$ & $1.57, \mathrm{~d}(12.7)$ & $1.43, \mathrm{~d}(12.9)$ & $1.53, \mathrm{~d}(12.8)$ & $1.49, \mathrm{~d}(12.7)$ \\
\hline $12 b$ & $0.97, \mathrm{~m}$ & $0.96, \mathrm{~m}$ & $0.86, \mathrm{~m}$ & 0.91, brd $(12.8)$ & $0.91, \mathrm{~m}$ \\
\hline $13 \alpha$ & $0.83,^{\mathrm{a}} \mathrm{s}$ & $0.83,{ }^{b} \mathrm{~s}$ & $0.75,{ }^{c} \mathrm{~s}$ & $0.79, \mathrm{~d}_{\mathrm{s}}$ & $0.80{ }^{\mathrm{f}} \mathrm{s}$ \\
\hline $14 \beta$ & $1.00,{ }^{a} \mathrm{~s}$ & $1.00, \mathrm{~b}_{\mathrm{s}}$ & $0.93,{ }^{c} \mathrm{~s}$ & $0.95, \mathrm{~d}_{\mathrm{s}}$ & $0.96,{ }^{\mathrm{f}} \mathrm{s}$ \\
\hline 15 & $0.94, \mathrm{~s}$ & $0.94, \mathrm{~s}$ & $0.86, \mathrm{~s}$ & $0.87, \mathrm{~s}$ & $0.92, \mathrm{~s}$ \\
\hline $1^{\prime} a, b$ & $3.56-3.43$ & $3.44-3.39$ & $3.63-3.53$ & $3.79-3.70$ & $3.70-3.62$ \\
\hline $2^{\prime}$ & $1.16, \mathrm{t}(7.0)$ & $1.51, \mathrm{~m}$ & $2.79, \mathrm{t}(7.2)$ & $4.03, \mathrm{t}(5.1)$ & $2.93, \mathrm{t}(6.4)$ \\
\hline $3^{\prime}$ & & $1.36, \mathrm{~m}$ & & & \\
\hline $4^{\prime}, 8^{\prime}$ & & $0.89, \mathrm{t}(7.3)$ & $7.23-7.10$ & $6.89-6.83^{e}$ & $7.38, \mathrm{~d}(8.7)$ \\
\hline $5^{\prime}, 7^{\prime}$ & & & $7.23-7.10$ & $7.23-7.17^{\mathrm{e}}$ & $8.12, \mathrm{~d}(8.7)$ \\
\hline $6^{\prime}$ & & & $7.23-7.10$ & $6.87, \mathrm{~m}$ & \\
\hline
\end{tabular}

${ }^{\mathrm{a}-\mathrm{f}}$ Interchangeable signals.

Table 3. ${ }^{13} \mathrm{C}$ NMR Spectroscopic Data $\left(\mathrm{CDCl}_{3}, 100 \mathrm{MHz}\right)$ for Compounds $\mathbf{2} \mathbf{b}, \mathbf{2} \mathbf{d}$, and $\mathbf{2} \mathbf{f}-\mathbf{2} \mathbf{h}$.

\begin{tabular}{|c|c|c|c|c|c|}
\hline Position & $\begin{array}{c}2 b \\
\delta_{C} \text {, Type }\end{array}$ & $\begin{array}{c}2 \mathrm{~d} \\
\delta_{\mathrm{C}}, \text { Type }\end{array}$ & $\begin{array}{c}2 f \\
\delta_{C}, \text { Type }\end{array}$ & $\begin{array}{c}2 \mathrm{~g} \\
\delta_{\mathrm{C}}, \text { Type }\end{array}$ & $\begin{array}{c}2 \mathrm{~h} \\
\delta_{\mathrm{C}}, \text { Type }\end{array}$ \\
\hline 1 & $44.1, \mathrm{C}$ & $44.3, \mathrm{C}$ & $44.3, \mathrm{C}$ & $44.3, \mathrm{C}$ & $44.3, \mathrm{C}$ \\
\hline 2 & 88.1, CH & 88.3, CH & $88.6, \mathrm{CH}$ & $89.1, \mathrm{CH}$ & $88.9, \mathrm{CH}$ \\
\hline 3 & $44.9, \mathrm{CH}_{2}$ & $44.7, \mathrm{CH}_{2}$ & $44.5, \mathrm{CH}_{2}$ & $44.6, \mathrm{CH}_{2}$ & $44.5, \mathrm{CH}_{2}$ \\
\hline 4 & $36.9, \mathrm{C}$ & $37.0, \mathrm{C}$ & $37.1, \mathrm{C}$ & $37.0, \mathrm{C}$ & $37.2, \mathrm{C}$ \\
\hline 5 & $50.6, \mathrm{CH}$ & $50.5, \mathrm{CH}$ & $50.5, \mathrm{CH}$ & $50.5, \mathrm{CH}$ & $50.4, \mathrm{CH}$ \\
\hline 6 & $20.5, \mathrm{CH}_{2}$ & 20.6, $\mathrm{CH}_{2}$ & 20.6, $\mathrm{CH}_{2}$ & 20.6, $\mathrm{CH}_{2}$ & 20.6, $\mathrm{CH}_{2}$ \\
\hline 7 & $33.1, \mathrm{CH}_{2}$ & $33.2, \mathrm{CH}_{2}$ & $33.1, \mathrm{CH}_{2}$ & $33.1, \mathrm{CH}_{2}$ & $33.1, \mathrm{CH}_{2}$ \\
\hline 8 & $34.7, \mathrm{C}$ & $34.7, \mathrm{C}$ & $34.7, \mathrm{C}$ & $34.7, \mathrm{C}$ & $34.6, \mathrm{C}$ \\
\hline 9 & $75.3, \mathrm{CH}$ & $75.3, \mathrm{CH}$ & $75.2, \mathrm{CH}$ & $75.2, \mathrm{CH}$ & $75.1, \mathrm{CH}$ \\
\hline 10 & 26.0, $\mathrm{CH}_{2}$ & $26.1, \mathrm{CH}_{2}$ & $26.0, \mathrm{CH}_{2}$ & 26.0, $\mathrm{CH}_{2}$ & $26.1, \mathrm{CH}_{2}$ \\
\hline 11 & 26.7, $\mathrm{CH}_{2}$ & 26.7, $\mathrm{CH}_{2}$ & 26.7, $\mathrm{CH}_{2}$ & 26.7, $\mathrm{CH}_{2}$ & $26.8, \mathrm{CH}_{2}$ \\
\hline 12 & $36.6, \mathrm{CH}_{2}$ & $36.5, \mathrm{CH}_{2}$ & $36.4, \mathrm{CH}_{2}$ & $36.4, \mathrm{CH}_{2}$ & $36.4, \mathrm{CH}_{2}$ \\
\hline 13 & $25.3,{ }^{\mathrm{a}} \mathrm{CH}_{3}$ & $25.4,{ }^{\mathrm{b}} \mathrm{CH}_{3}$ & $25.4,{ }^{\mathrm{c}} \mathrm{CH}_{3}$ & $25.4{ }^{\mathrm{d}} \mathrm{CH}_{3}$ & $25.4,{ }^{\mathrm{f}} \mathrm{CH}_{3}$ \\
\hline 14 & $31.2,{ }^{\mathrm{a}} \mathrm{CH}_{3}$ & $31.3, \mathrm{~b} \mathrm{CH}_{3}$ & $31.3,{ }^{\mathrm{c}} \mathrm{CH}_{3}$ & $31.3,{ }^{\mathrm{d}} \mathrm{CH}_{3}$ & $31.3,{ }^{\mathrm{f}} \mathrm{CH}_{3}$ \\
\hline 15 & $28.4, \mathrm{CH}_{3}$ & $28.4, \mathrm{CH}_{3}$ & $28.4, \mathrm{CH}_{3}$ & $28.4, \mathrm{CH}_{3}$ & 28.3, $\mathrm{CH}_{2}$ \\
\hline $1^{\prime}$ & $65.8, \mathrm{CH}_{2}$ & $70.3, \mathrm{CH}_{2}$ & $71.6, \mathrm{CH}_{2}$ & $68.9, \mathrm{CH}_{2}$ & $70.2, \mathrm{CH}_{2}$ \\
\hline $2^{\prime}$ & $15.7, \mathrm{CH}_{3}$ & $32.3, \mathrm{CH}_{2}$ & $36.9, \mathrm{CH}_{2}$ & $67.6, \mathrm{CH}_{2}$ & $36.7, \mathrm{CH}_{2}$ \\
\hline $3^{\prime}$ & & $19.4, \mathrm{CH}_{2}$ & $139.4, \mathrm{C}$ & $158.9, \mathrm{C}$ & $147.8, \mathrm{C}$ \\
\hline $4^{\prime}, 8^{\prime}$ & & $14.0, \mathrm{CH}_{3}$ & $129.0,2 \mathrm{CH}$ & 114.7, e $2 \mathrm{CH}$ & $129.9,2 \mathrm{CH}$ \\
\hline $5^{\prime}, 7^{\prime}$ & & & $128.2,2 \mathrm{CH}$ & 129.3 , e $2 \mathrm{CH}$ & $123.3,2 \mathrm{CH}$ \\
\hline $6^{\prime}$ & & & 126.0, $\mathrm{CH}$ & $120.7, \mathrm{CH}$ & $146.5, \mathrm{C}$ \\
\hline
\end{tabular}

${ }^{\mathrm{a}-\mathrm{f}}$ Interchangeable signals. 
(2S,9R)-2-butoxyclovan-9-ol (2d): needles $\left(\mathrm{CHCl}_{3}\right) ; 50-52{ }^{\circ} \mathrm{C} ;[\alpha]_{D}^{25}+10.0$ (c 3.8, $\left.\mathrm{CHCl}_{3}\right)$; IR (film) $v_{\max } 3406,2927,2864,1463,1361,1106 \mathrm{~cm}^{-1} ;{ }^{1} \mathrm{H} \mathrm{NMR}$ data $\left(\mathrm{CDCl}_{3}, 400 \mathrm{MHz}\right)$, see Table $2 ;{ }^{13} \mathrm{C} \mathrm{NMR}$ data $\left(\mathrm{CDCl}_{3}, 100 \mathrm{MHz}\right)$, see Table 3; HMBC (selected correlations) $\mathrm{C}-1 \rightarrow \mathrm{H}-2 \alpha, \mathrm{H}-3 \alpha, \mathrm{H}-3 \beta, \mathrm{H}-6 \mathrm{a}, \mathrm{H}-6 \mathrm{~b}, \mathrm{H}-11 \mathrm{a}, \mathrm{H}-12 \mathrm{a} ; \mathrm{C}-2 \rightarrow \mathrm{H}-3 \alpha, \mathrm{H}-3 \beta, \mathrm{H}-12 \mathrm{~b}, \mathrm{H}-1^{\prime} \mathrm{a}, \mathrm{b}$; $\mathrm{C}-4 \rightarrow \mathrm{H}-3 \alpha, \mathrm{H}-3 \beta, \mathrm{H}-13 \alpha, \mathrm{H}-14 \beta$; C-8 $\rightarrow$ H-7a, H-12a, H-15; C-15 $\rightarrow$ H-7a, H-7b, H-12a, $\mathrm{H}-12 \mathrm{~b} ; \mathrm{C}-1^{\prime} \rightarrow, \mathrm{H}-2 \alpha, \mathrm{H}_{2}-2^{\prime}, \mathrm{H}_{2}-3^{\prime} ; \mathrm{C}-2^{\prime} \rightarrow \mathrm{H}-1^{\prime} \mathrm{a}, \mathrm{b}, \mathrm{H}_{2}-3^{\prime}, \mathrm{H}_{3}-4^{\prime} ; \mathrm{C}-3^{\prime} \rightarrow \mathrm{H}-1^{\prime} \mathrm{a}, \mathrm{b}, \mathrm{H}_{2}-2^{\prime}$, $\mathrm{H}_{3}-4^{\prime}$; C-4' $\rightarrow \mathrm{H}_{2}-2^{\prime}, \mathrm{H}_{2}-3^{\prime}$; EIMS m/z 294 [M] ${ }^{+}$(3), 279 (3), 220 (7), 205 (7), 187 (5), 161 (9), 141 (100), 121 (7), 105 (13), 95 (10), 85 (44); HREIMS m/z 294.2520 [M] $^{+}$(calcd for $^{2}$ $\left.\mathrm{C}_{19} \mathrm{H}_{34} \mathrm{O}_{2}, 294.2559\right)$.

(2S,9R)-2-(2'-phenylethoxy)clovan-9-ol (2f): needles $\left(\mathrm{CHCl}_{3}\right) ; 78-80{ }^{\circ} \mathrm{C} ;[\alpha]_{D}^{25}+16(\mathrm{c} 3.4$, $\left.\mathrm{CDCl}_{3}\right)$; IR (film) $v_{\max } 3526,2926,2862,1456,1106,750,698 \mathrm{~cm}^{-1} ;{ }^{1} \mathrm{H} \mathrm{NMR}$ data $\left(\mathrm{CDCl}_{3}\right.$, $400 \mathrm{MHz})$, see Table $2 ;{ }^{13} \mathrm{C}$ NMR data $\left(\mathrm{CDCl}_{3}, 100 \mathrm{MHz}\right)$, see Table 3; HMBC (selected correlations) C- $1 \rightarrow \mathrm{H}-2 \alpha, \mathrm{H}-3 \alpha, \mathrm{H}-12 \mathrm{a}, \mathrm{H}-12 \mathrm{~b} ; \mathrm{C}-2 \rightarrow \mathrm{H}-3 \alpha, \mathrm{H}-3 \beta, \mathrm{H}-1^{\prime} \mathrm{a}, \mathrm{b} ; \mathrm{C}-15 \rightarrow \mathrm{H}-7 \mathrm{a}$, $\mathrm{H}-7 \mathrm{~b}, \mathrm{H}-12 \mathrm{a} ; \mathrm{C}-1^{\prime} \rightarrow \mathrm{H}-2 \alpha, \mathrm{H}_{2}-2^{\prime} ; \mathrm{C}-2^{\prime} \rightarrow \mathrm{H}-1^{\prime} \mathrm{a}, \mathrm{b}, \mathrm{H}-4^{\prime}, \mathrm{H}-8^{\prime} ; \mathrm{C}-3^{\prime} \rightarrow \mathrm{H}-1^{\prime} \mathrm{a}, \mathrm{b}, \mathrm{H}_{2}-2^{\prime} ; \mathrm{C}-4^{\prime}$, $\mathrm{C}-8^{\prime} \rightarrow \mathrm{H}_{2}-2^{\prime}$; HRESIMS $m / z$ 365.2467 [M+Na] ${ }^{+}$(calcd for $\mathrm{C}_{23} \mathrm{H}_{34} \mathrm{O}_{2} \mathrm{Na}, 365.2453$ ), $m / z$ $325.2521\left[\mathrm{M}+\mathrm{H}-\mathrm{H}_{2} \mathrm{O}\right]^{+}$(calcd for $\mathrm{C}_{23} \mathrm{H}_{33} \mathrm{O}, 325.2531$ ), $m / z 221.1890\left[\mathrm{M}+\mathrm{H}-\mathrm{C}_{8} \mathrm{H}_{10} \mathrm{O}\right]^{+}$(calcd for $\left.\mathrm{C}_{15} \mathrm{H}_{25} \mathrm{O}, 221.1905\right)$.

(2S,9R)-2-(2'-phenoxyethoxy)clovan-9-ol (2g): yellow oil; $[\alpha]_{D}^{25}+5$ (c 3.4, $\left.\mathrm{CDCl}_{3}\right)$; IR (film) $v_{\max } 3436,2928,2864,1600,1496,752,690 \mathrm{~cm}^{-1} ;{ }^{1} \mathrm{H}$ NMR data $\left(\mathrm{CDCl}_{3}, 400 \mathrm{MHz}\right)$, see Table $2 ;{ }^{13} \mathrm{C}$ NMR data $\left(\mathrm{CDCl}_{3}, 100 \mathrm{MHz}\right)$, see Table 3; HMBC (selected correlations) $\mathrm{C}-1 \rightarrow \mathrm{H}-3 \alpha, \mathrm{H}-3 \beta, \mathrm{H}-5 \beta, \mathrm{H}-12 \mathrm{a}, \mathrm{H}-12 \mathrm{~b} ; \mathrm{C}-2 \rightarrow \mathrm{H}-3 \alpha, \mathrm{H}-3 \beta, \mathrm{H}-1^{\prime} \mathrm{a}, \mathrm{b} ; \mathrm{C}-15 \rightarrow \mathrm{H}-7 \mathrm{a}, \mathrm{H}-7 \mathrm{~b}$, $\mathrm{H}-12 \mathrm{a} ; \mathrm{C}-1^{\prime} \rightarrow \mathrm{H}-2 \alpha, \mathrm{H}_{2}-2^{\prime}$; C-2 $\rightarrow$ H-1'a,b; C-3 ${ }^{\prime} \rightarrow \mathrm{H}_{2}-2^{\prime}, \mathrm{H}-4^{\prime}, \mathrm{H}-8^{\prime}, \mathrm{H}-5^{\prime}, \mathrm{H}^{\prime} 7^{\prime}$; HRESIMS $m / z 381.2404[\mathrm{M}+\mathrm{Na}]^{+}$(calcd for $\mathrm{C}_{23} \mathrm{H}_{34} \mathrm{O}_{3} \mathrm{Na}, 381.2406$ ), $m / z 341.2505\left[\mathrm{M}+\mathrm{H}_{-} \mathrm{H}_{2} \mathrm{O}\right]^{+}$(calcd for $\mathrm{C}_{23} \mathrm{H}_{33} \mathrm{O}_{2}, 341.2481$ ), $m / z 221.1898$ [M+H- $\left.\mathrm{C}_{8} \mathrm{H}_{10} \mathrm{O}_{2}\right]^{+}$(calcd for $\mathrm{C}_{15} \mathrm{H}_{25} \mathrm{O}, 221.1905$ ).

${ }^{1} \mathrm{H}-\mathrm{NMR}$ and ${ }^{13} \mathrm{C}-\mathrm{NMR}$ spectra can be found as Supplementary Materials for compounds $\mathbf{2 b}, \mathbf{2 d}, \mathbf{2 f}$ and $\mathbf{2 g}$ (Figures S1-S8 and S1a-S8a).

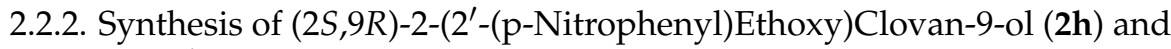
$(2 S, 9 R)-2-\left(2^{\prime}-(\right.$ p-Nitrophenoxy)Ethoxy)Clovan-9-ol (2i)

We dissolved $500 \mathrm{mg}(2.27 \mathrm{mmol})$ of (-)-caryophyllene oxide by stirring in a solution of either 2-(p-nitrophenyl)ethanol (500 mg, $2.99 \mathrm{mmol})$ or 2-(p-nitrophenoxy)ethanol (600 mg, $3.27 \mathrm{mmol})$ in propan-2-one $(5 \mathrm{~mL})$ at room temperature. Then, $60 \mathrm{mg}(0.47 \mathrm{mmol})$ of TCNE was added and the reaction was monitored by TLC. After $21 \mathrm{~h}$ the solvent was evaporated under reduced pressure. Purification by column chromatography of the crude reaction mixture on silica gel, eluted with increasing gradients of ethyl acetate in petroleum ether, and combined with HPLC purification, yielded compound $2 \mathrm{~h}$ (54 $\mathrm{mg}, 0.14 \mathrm{mmol}, 6 \%$ ) or compound $2 \mathrm{i}$ [38] (64 mg, $0.16 \mathrm{mmol}, 7 \%$; see revised assignment of ${ }^{1} \mathrm{H}$ and ${ }^{13} \mathrm{C} \mathrm{NMR}$ spectra and selected HMBC correlations in the Supporting Materials section, Table S1) (Figure 2).

(2S,9R)-2-(2'-(p-nitrophenyl)ethoxy)clovan-9-ol (2h): yellow oil; $[\alpha]_{D}^{25}+26$ (c 3.4, $\left.\mathrm{CDCl}_{3}\right)$; IR (film) $v_{\max } 3430,2946,2926,2860,1510,1344,1108,852 \mathrm{~cm}^{-1} ;{ }^{1} \mathrm{H} \mathrm{NMR}\left(\mathrm{CDCl}_{3}\right.$, $400 \mathrm{MHz})$, see Table $2 ;{ }^{13} \mathrm{C}$ NMR $\left(\mathrm{CDCl}_{3}, 100 \mathrm{MHz}\right)$, see Table 3; HMBC (selected correlations): C-1 $\rightarrow \mathrm{H}-2 \alpha, \mathrm{H}-3 \alpha, \mathrm{H}-12 \mathrm{a} ; \mathrm{C}-2 \rightarrow \mathrm{H}-3 \alpha, \mathrm{H}-3 \beta, \mathrm{H}-5 \beta, \mathrm{H}-12 \mathrm{~b}, \mathrm{H}-1^{\prime} \mathrm{a}, \mathrm{b} ; \mathrm{C}-9 \rightarrow \mathrm{H}_{3}-15 \beta$, $\mathrm{H}-7 \mathrm{a} ; \mathrm{C}-15 \rightarrow \mathrm{H}-7 \mathrm{a}, \mathrm{H}-7 \mathrm{~b} ; \mathrm{C}-1^{\prime} \rightarrow \mathrm{H}-2 \alpha, \mathrm{H}_{2}-2^{\prime}$; C-2 ${ }^{\prime} \rightarrow \mathrm{H}-1^{\prime} \mathrm{a}, \mathrm{b}, \mathrm{H}-4^{\prime}, \mathrm{H}-8^{\prime} ; \mathrm{C}-3^{\prime} \rightarrow \mathrm{H}-1^{\prime} \mathrm{a}, \mathrm{b}$, $\mathrm{H}_{2}-2^{\prime}, \mathrm{H}-5^{\prime}, \mathrm{H}-7^{\prime} ; \mathrm{C}-4^{\prime}, \mathrm{C}-8^{\prime} \rightarrow \mathrm{H}_{2}-2^{\prime} ; \mathrm{C}-6^{\prime} \rightarrow \mathrm{H}^{\prime} 4^{\prime}, \mathrm{H}-8^{\prime} ; \mathrm{HRESIMS} m / z$ 410.2300 [M+Na] ${ }^{+}$ (calcd for $\mathrm{C}_{23} \mathrm{H}_{33} \mathrm{NO}_{4} \mathrm{Na}, 410.2307$ ), $m / z$ 370.2388 [M+H-H $\left.\mathrm{H}_{2} \mathrm{O}\right]^{+}$(calcd for $\mathrm{C}_{23} \mathrm{H}_{32} \mathrm{NO}_{3}$, 370.2382), $m / z$ 203.1813 [M+H- $\left.{ }_{8} \mathrm{H}_{9} \mathrm{NO}_{3}-\mathrm{H}_{2} \mathrm{O}\right]^{+}$(calcd for $\mathrm{C}_{15} \mathrm{H}_{23}, 203.1800$ ).

${ }^{1} \mathrm{H}-\mathrm{NMR}$ and ${ }^{13} \mathrm{C}-\mathrm{NMR}$ spectra can be found as Supplementary Materials for compounds $\mathbf{2 h}$ and $\mathbf{2 i}$ (Figures S9-S12 and S9a-S12a).

\subsection{General Procedure for the Alcoholysis of (1R,5R,9S)-Caryophylla-4(12),8(13)-Diene-5-ol} Catalyzed by Tin(II) Triflate

$(1 R, 5 R, 9 S)$-caryophylla-4(12),8(13)-diene-5-ol (3) (250 mg, $1.14 \mathrm{mmol}$ ) dissolved in the alcohol $(5 \mathrm{~mL})$ was treated with a catalytic amount of $\mathrm{Sn}(\mathrm{OTf})_{2}(250 \mathrm{mg}, 1.14 \mathrm{mmol})$ 
at room temperature under an argon atmosphere. After $24 \mathrm{~h}$, once compound 3 was consumed, as shown by TLC monitoring, the solvent was either evaporated under vacuum or washed with brine. The resulting gum was redissolved in ethyl acetate and dried over anhydrous $\mathrm{Na}_{2} \mathrm{SO}_{4}$. After filtration, evaporation of the solvent afforded a crude reaction product. Purification by column chromatography on silica gel, with increasing gradients of ethyl acetate in petroleum ether, combined with HPLC purification, yielded 4a [36], $\mathbf{4 b}$ [49], $\mathbf{4} \mathbf{c}-\mathbf{4} \mathbf{e}$, as well as rearrangement compounds (8R,9R)-isocaryolan-9-one (5) [36] and $(1 S, 2 S, 5 R, 8 S)$-8-methylene-1,4,4-trimethyltricyclo [6.2.1.0 $\left.{ }^{2,5}\right]$ undecane-8-carbaldehyde (6) [36] (Figure 3); (reaction conditions, products and yields in Table 4).<smiles>C=C1CC[C@@H]2O[C@]2(C)CC[C@H]2[C@@H](C)C[C@H]1C2(C)Br</smiles>

Caryophyllene oxide<smiles>C=C1CC[C@@H]2C(=C)CC[C@H]2C(=C)C[C@@H]1O</smiles>

3

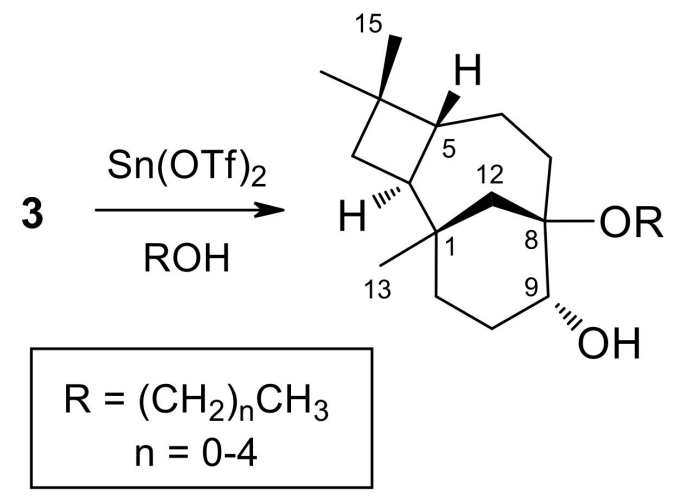

$$
\begin{aligned}
& \text { 4a } \mathrm{R}=\mathrm{CH}_{3} \\
& \text { 4b } \mathrm{R}=\left(\mathrm{CH}_{2}\right) \mathrm{CH}_{3} \\
& \text { 4c } \mathrm{R}=\left(\mathrm{CH}_{2}\right)_{2} \mathrm{CH}_{3} \\
& \text { 4d } \mathrm{R}=\left(\mathrm{CH}_{2}\right)_{3} \mathrm{CH}_{3} \\
& \text { 4e } \mathrm{R}=\left(\mathrm{CH}_{2}\right)_{4} \mathrm{CH}_{3}
\end{aligned}
$$

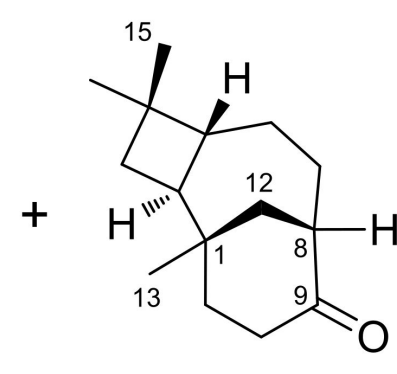

5

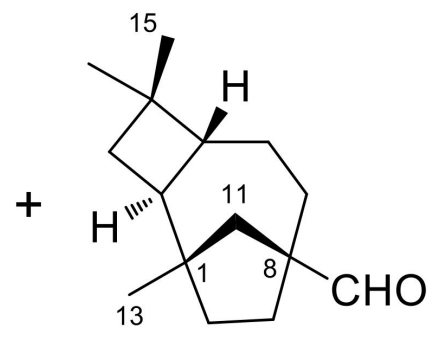

6

Figure 3. Synthesis of 2-Alkoxyisocaryolan-9-ols (4a-4e).

Table 4. Alcoholysis of Caryophyllene Oxide Catalyzed by Sn(OTf $)_{2}$. Reaction Conditions, Products and Yields.

\begin{tabular}{cc}
\hline Alcohol & Products (Yield) \\
\hline $\mathrm{CH}_{3} \mathrm{OH}$ & $\mathbf{4 a}(42 \%), \mathbf{5}(13 \%), \mathbf{6}(27 \%)$ \\
$\mathrm{CH}_{3} \mathrm{CH}_{2} \mathrm{OH}$ & $4 \mathrm{~b}(39 \%), \mathbf{5}(14 \%), \mathbf{6}(29 \%)$ \\
$\mathrm{CH}_{3}\left(\mathrm{CH}_{2}\right)_{2} \mathrm{OH}$ & $4 \mathrm{c}(35 \%), \mathbf{5}(16 \%), \mathbf{6}(31 \%)$ \\
$\mathrm{CH}_{3}\left(\mathrm{CH}_{2}\right)_{3} \mathrm{OH}$ & $4 \mathrm{~d}(34 \%), \mathbf{5}(19 \%), \mathbf{6}(42 \%)$ \\
$\mathrm{CH}_{3}\left(\mathrm{CH}_{2}\right)_{4} \mathrm{OH}$ & $4 \mathrm{e}(35 \%), \mathbf{5}(20 \%), \mathbf{6}(37 \%)$ \\
\hline
\end{tabular}




\section{Spectroscopic Data of Compounds $4 \mathrm{c}-4 \mathrm{e}$}

(8R,9R)-8-propoxyisocaryolan-9-ol (4c): oil; $[\alpha]_{D}^{25}-4$ (c 3.4, $\mathrm{CHCl}_{3}$ ); IR (film) $v_{\max } 3435$, 2947, 1458, 1078, $559 \mathrm{~cm}^{-1} ;{ }^{1} \mathrm{H}$ NMR (CDCl $\left.3,400 \mathrm{MHz}\right)$, see Table $5 ;{ }^{13} \mathrm{C} \mathrm{NMR}\left(\mathrm{CDCl}_{3}\right.$, $100 \mathrm{MHz}$ ), see Table 5; HMBC (selected correlations): C-8 $\rightarrow$ H-9 $\beta, \mathrm{H}-12 \mathrm{a}, \mathrm{H}-12 \mathrm{~b}$; $\mathrm{C}-1^{\prime} \rightarrow \mathrm{H}_{2}-2^{\prime}, \mathrm{H}_{3}-3^{\prime} ; \mathrm{C}-2^{\prime} \rightarrow \mathrm{H}-1^{\prime} \mathrm{a}, \mathrm{b}, \mathrm{H}_{3}-3^{\prime} ; \mathrm{C}-3^{\prime} \rightarrow \mathrm{H}-1^{\prime} \mathrm{a}, \mathrm{b}, \mathrm{H}_{2}-2^{\prime} ;$ EIMS $m / z 280[\mathrm{M}]^{+}(2)$, 230 (1), 221 (100), 168 (3) 164 (5), 130 (6), 108 (10); HREIMS $m / z 280.2386$ [M] (calcd for $\left.\mathrm{C}_{18} \mathrm{H}_{32} \mathrm{O}_{2}, 280.2402\right)$.

Table 5. NMR Spectroscopic Data for Compounds $4 \mathbf{c}-\mathbf{4 e}$ in $\mathrm{CDCl}_{3}(\mathrm{~J}$ in $\mathrm{Hz})$.

\begin{tabular}{|c|c|c|c|c|c|c|}
\hline & $4 c$ & & $4 d$ & & $4 e$ & \\
\hline Position & $\delta_{\mathrm{H}}(400 \mathrm{MHz})$ & $\begin{array}{c}\delta_{\mathrm{C}}(100 \mathrm{MHz}), \\
\text { Type }\end{array}$ & $\delta_{\mathrm{H}}(400 \mathrm{MHz})$ & $\begin{array}{c}\delta_{\mathrm{C}}(100 \mathrm{MHz}), \\
\text { Type }\end{array}$ & $\delta_{\mathrm{H}}(400 \mathrm{MHz})$ & $\begin{array}{c}\delta_{\mathrm{C}}(100 \mathrm{MHz}), \\
\text { Type }\end{array}$ \\
\hline 1 & & $32.83, \mathrm{C}$ & & $32.83,{ }^{\mathrm{a}} \mathrm{C}$ & & $32.88, \mathrm{C}$ \\
\hline $2 \alpha$ & $2.08, \operatorname{ddd}(11.9,10.3,8.0)$ & $36.57, \mathrm{CH}$ & 2.07, ddd $(12.4,11.8,7.9)$ & $36.55, \mathrm{CH}$ & 2.07, ddd $(12.0,10.5,7.9)$ & $36.55, \mathrm{CH}$ \\
\hline $3 \alpha$ & $1.44, \mathrm{~m}$ & & $1.45, \mathrm{~m}$ & $3552 \mathrm{CH}_{2}$ & $1.44, \mathrm{dd}(9.8,7.9)$ & $3551 \mathrm{CH}_{2}$ \\
\hline $3 \beta$ & $1.27, \mathrm{t}(10.3)$ & $35.52, \mathrm{CH}_{2}$ & $1.27, \mathrm{t}(10.2)$ & $35.52, \mathrm{CH}_{2}$ & $1.26, \mathrm{~m}$ & $35.51, \mathrm{CH}_{2}$ \\
\hline 4 & & $34.88, \mathrm{C}$ & & $34.88, \mathrm{C}$ & & $34.87, \mathrm{C}$ \\
\hline $5 \beta$ & $1.72, \mathrm{~m}$ & $43.96, \mathrm{CH}$ & $1.71, \mathrm{~m}$ & $43.95, \mathrm{CH}$ & $1.70, \mathrm{~m}$ & $43.94, \mathrm{CH}$ \\
\hline $6 a$ & $1.50, \mathrm{~m}$ & & $1.48, \mathrm{~m}$ & & $1.49, \mathrm{~m}$ & \\
\hline $6 b$ & $1.61, \mathrm{~m}$ & $21.70, \mathrm{CH}_{2}$ & $1.61, \mathrm{~m}$ & $21.71, \mathrm{CH}_{2}$ & $1.61, \mathrm{~m}$ & $21.72, \mathrm{CH}_{2}$ \\
\hline $7 a$ & $1.53, \mathrm{~m}$ & & $1.52, \mathrm{~m}$ & $29.29 . \mathrm{CH}_{2}$ & $1.53, \mathrm{~m}$ & \\
\hline $7 \mathrm{~b}$ & $1.87, \mathrm{~m}$ & $29.29, \mathrm{CH}_{2}$ & $1.86, \mathrm{~m}$ & $29.29, \mathrm{CH}_{2}$ & $1.86, \mathrm{~m}$ & $29.20, C_{2}$ \\
\hline 8 & & $79.96, \mathrm{C}$ & & $79.95, \mathrm{C}$ & & $79.96, \mathrm{C}$ \\
\hline $9 \beta$ & 3.56, dd $(10.8,6.3)$ & $76.86, \mathrm{CH}$ & 3.55, dd $(11.4,5.8)$ & $76.85, \mathrm{CH}$ & 3.55, dd $(11,3,5.8)$ & $76.82, \mathrm{CH}$ \\
\hline $10 \mathrm{a}$ & $1.84-1.70, \mathrm{~m}$ & & $1.83-1.70, \mathrm{~m}$ & & $1.82-1.70, \mathrm{~m}$ & \\
\hline $10 \mathrm{~b}$ & $1.84-1.70, \mathrm{~m}$ & $27.09, \mathrm{CH}_{2}$ & $1.83-1.70, \mathrm{~m}$ & $27.08, \mathrm{CH}_{2}$ & $1.82-1.70, \mathrm{~m}$ & $27.10, \mathrm{CH}_{2}$ \\
\hline $11 \alpha$ & $1.21, \mathrm{td}(13.2,5.3)$ & & $1.21, \mathrm{td}(12.8,4.9)$ & & $1.20, \mathrm{td}(13.6,5.1)$ & \\
\hline $11 \beta$ & $1.37, \mathrm{~m}$ & $36.65, \mathrm{CH}_{2}$ & $1.37, \mathrm{~m}$ & $36.64, \mathrm{CH}_{2}$ & $1.36, \mathrm{~m}$ & $36.63, \mathrm{CH}_{2}$ \\
\hline $12 \mathrm{a}$ & 1.87, brd (13.0) & & 1.86, brd $(12.5)$ & & $1.87, \mathrm{~m}$ & \\
\hline $12 b$ & $0.89, \mathrm{~d}(13.0)$ & $42.70, \mathrm{CH}_{2}$ & $0.91, \mathrm{~d}(12.5)$ & $42.6 \%, \mathrm{CH}_{2}$ & $0.90, \mathrm{~m}$ & $42.6 \%, \mathrm{CH}_{2}$ \\
\hline 13 & $0.97, \mathrm{~s}$ & $26.24, \mathrm{CH}_{3}$ & $0.97, \mathrm{~s}$ & $26.24, \mathrm{CH}_{3}$ & $0.96, \mathrm{~s}$ & $26.23, \mathrm{CH}_{3}$ \\
\hline $14 \alpha$ & $0.96, \mathrm{~s}$ & $20.84, \mathrm{CH}_{3}$ & $0.96, \mathrm{~s}$ & $20.84, \mathrm{CH}_{3}$ & $0.95, \mathrm{~s}$ & $20.83, \mathrm{CH}_{3}$ \\
\hline $15 \beta$ & $0.79 \mathrm{~s}$ & $30.67, \mathrm{CH}_{3}$ & $0.79, \mathrm{~s}$ & $30.67, \mathrm{CH}_{3}$ & $0.78, \mathrm{~s}$ & $30.66, \mathrm{CH}_{3}$ \\
\hline $1^{\prime} a, b$ & $3.40-3.28$ & $62.68, \mathrm{CH}_{2}$ & $3.44-3.31$ & $60.68, \mathrm{CH}_{2}$ & $3.43-3.30$ & $61.03, \mathrm{CH}_{2}$ \\
\hline $2^{\prime}$ & $1.49, \mathrm{~m}$ & $23.93, \mathrm{CH}_{2}$ & $1.44, \mathrm{~m}$ & $32.85,{ }^{\mathrm{a}} \mathrm{CH}_{2}$ & $1.46, \mathrm{~m}$ & $30.45, \mathrm{CH}_{2}$ \\
\hline $3^{\prime}$ & $0.88, \mathrm{t}(7.4)$ & $10.78, \mathrm{CH}_{3}$ & $1.33, \mathrm{~m}$ & $19.47, \mathrm{CH}_{2}$ & $1.27, \mathrm{~m}$ & $28.50, \mathrm{CH}_{2}$ \\
\hline $4^{\prime}$ & & & $0.89, \mathrm{t}(7.4)$ & $13.96, \mathrm{CH}_{3}$ & $1.29, \mathrm{~m}$ & $22.56, \mathrm{CH}_{2}$ \\
\hline $5^{\prime}$ & & & & & $0.89, \mathrm{t}(7.0)$ & $14.07, \mathrm{CH}_{3}$ \\
\hline
\end{tabular}

\footnotetext{
a Interchangeable signals.
}

(8R,9R)-8-butoxyisocaryolan-9-ol (4d): oil; $[\alpha]_{D}^{25}-4$ (c 4.1, $\mathrm{CHCl}_{3}$ ); IR (film) $v_{\max } 3334$, 2945, 1458, 1080, $559 \mathrm{~cm}^{-1} ;{ }^{1} \mathrm{H}$ NMR $\left(\mathrm{CDCl}_{3}, 400 \mathrm{MHz}\right)$, see Table $5 ;{ }^{13} \mathrm{C} \mathrm{NMR}\left(\mathrm{CDCl}_{3}\right.$, $100 \mathrm{MHz}$ ), see Table 5; HMBC (selected correlations): C- $8 \rightarrow \mathrm{H}-9 \beta, \mathrm{H}-1^{\prime} \mathrm{a}, \mathrm{H}-1^{\prime} \mathrm{b}, \mathrm{H}-12 \mathrm{a}$, $\mathrm{H}-12 \mathrm{~b} ; \mathrm{C}-1^{\prime} \rightarrow \mathrm{H}_{2}-2^{\prime}, \mathrm{H}_{2}-3^{\prime}$; C-2' $\rightarrow \mathrm{H}-1^{\prime} \mathrm{a}, \mathrm{b}, \mathrm{H}_{2}-3^{\prime} ; \mathrm{C}-3^{\prime} \rightarrow \mathrm{H}-1^{\prime} \mathrm{a}, \mathrm{b}, \mathrm{H}_{2}-2^{\prime} ; \mathrm{C}-4^{\prime} \rightarrow \mathrm{H}_{2}-2^{\prime}$, $\mathrm{H}_{2}-3^{\prime}$; EIMS m/z $294\left[\mathrm{M}^{+}\right.$(3), 279 (3), 243 (6), 236 (42), 235 (100), 179 (26), 163 (19), 135 (11), 107 (18), 68 (16), 55 (8); HREIMS $m / z 294.2592$ [M] (calcd for $\mathrm{C}_{19} \mathrm{H}_{34} \mathrm{O}_{2}, 294.2559$ ).

(8R,9R)-8-pentoxyisocaryolan-9-ol (4e): oil; $[\alpha]_{D}^{25}-2$ (c 4.9, $\mathrm{CHCl}_{3}$ ); IR (film) $v_{\max } 3433$, 2940, 1457, 1085, $560 \mathrm{~cm}^{-1} ;{ }^{1} \mathrm{H}$ NMR $\left(\mathrm{CDCl}_{3}, 400 \mathrm{MHz}\right)$, see Table $5 ;{ }^{13} \mathrm{C} \mathrm{NMR}\left(\mathrm{CDCl}_{3}\right.$, $100 \mathrm{MHz}$ ), see Table 5; HMBC (selected correlations): C- $8 \rightarrow \mathrm{H}-9 \beta, \mathrm{H}-12 \mathrm{a}, \mathrm{H}-12 \mathrm{~b}$; $\mathrm{C}-1^{\prime} \rightarrow \mathrm{H}_{2}-2^{\prime}, \mathrm{H}_{2}-3^{\prime}$; C-2' $\rightarrow$ H-1'a,b, $\mathrm{H}_{2}-3^{\prime}$; C-3' $\rightarrow$ H-1'a,b, $\mathrm{H}_{2}-2^{\prime}$; EIMS $m / z 308[\mathrm{M}]^{+}$ (15), 292 (12), 237 (16), 218 (20), 180 (14), 164 (28), 141 (60), 93 (26), 71 (100), 68 (2), 43 (42); HREIMS $m / z$ 308.2733 [M] $]^{+}$(calcd for $\mathrm{C}_{20} \mathrm{H}_{36} \mathrm{O}_{2}, 308.2715$ ).

${ }^{1} \mathrm{H}-\mathrm{NMR}$ and ${ }^{13} \mathrm{C}-\mathrm{NMR}$ spectra can be found as Supplementary Materials for compounds $\mathbf{4 b - 4 e}$ (Figures S13-S20 and S13a-S20a).

\subsection{Microorganism and Antifungal Assays}

The culture of the B. cinerea strain UCA 992 employed in this work was isolated from Domecq vineyard grapes, Jerez de la Frontera, Cádiz, Spain. This culture of B. cinerea has been deposited at the Mycological Herbarium Collection (UCA), Facultad de Ciencias, Universidad de Cádiz. Antifungal bioassays were performed by measuring radial growth 
on agar medium in a Petri dish in the presence of test compounds. Test compounds were dissolved in $\mathrm{EtOH}$ to a final compound concentration in the culture medium of $10^{-4} \mathrm{M}$. Solutions of test compounds were added to glucose-malt-peptone-agar medium (61 g of glucose-malt-peptone-agar per L, pH 6.5-7.0). The final EtOH concentration was identical in both the control and treated cultures. The medium was poured into 6 or $9 \mathrm{~cm}$ diameter sterile plastic Petri dishes and a mycelia disk of B. cinerea, $5 \mathrm{~mm}$ in diameter and cut from an actively growing culture, was placed in the center of the agar plate. Radial growth was measured for five days. The fungal growth inhibition percentage was calculated as $((\mathrm{D} 1-\mathrm{D} 2) / \mathrm{D} 1) \times 100$, where D1 is the average diameter of the control colony and D2 is the average diameter of the treated colony. Every concentration was evaluated in triplicate. Fungal growth inhibition percentages are given as averages +/ - standard deviations

\subsection{Bioassay for the Germination and Growth of Lettuce Seeds}

L. sativa (cv. Grand Rapids) seeds were purchased from Isla Pak, RS, Brazil. All undersized and damaged seeds were discarded. The bioassay was conducted in $100 \mathrm{~mm}$ Petri dishes containing Whatman \#1 filter paper $(90 \mathrm{~mm})$ as a support. L. sativa seeds (25 per dish), controls, and test compound solutions $(10 \mathrm{~mL})$ at concentrations of $10^{-3}$ and $10^{-5} \mathrm{M}$ were placed in the dishes. All solutions were prepared with deionized water and the $\mathrm{pH}$ values, buffered with $10 \mathrm{mM}$ 2-(N-morpholino)ethanesulfonic acid (MES), were adjusted to 6.0-6.5 with $\mathrm{NaOH}$ solution. Concentrations lower than $10^{-3} \mathrm{M}$ were obtained by serial dilution. Dishes were wrapped with Parafilm to reduce evaporation and incubated in the dark at $25^{\circ} \mathrm{C}$ in an environmental chamber. After 5 days, germinated seeds were counted (a seed was considered to be germinated when the radicle was at least $0.2 \mathrm{~mm}$ long) and the lengths of the radicle and shoots were measured using a pachymeter [43]. Dishes were kept at $4{ }^{\circ} \mathrm{C}$ during the measurement process to prevent subsequent growth. Osmotic pressure values were measured on a micro-osmometer and ranged between 30 and 38 mOsmolar [45]. The experiments were carried out in triplicate.

The effects of the test compounds on L. sativa germination and growth are given as percentage differences from the control, calculated from the differences (in $\mathrm{cm}$ ) between mean values obtained upon the addition of test compounds and mean values obtained for the control: (seeds grown without addition of tested compounds)/mean values for control $\times 100$. Thus, zero represents the control, positive values represent stimulation of the studied parameter, and negative values represent inhibition. The data were evaluated using Student's $t$-tests and the differences between the experiment and control were significant at a value of $p \leq 0.0517$.

\section{Results and Discussion}

The chemical transformations carried out for the preparation of 2-alkoxyclovan-9-ols (2a-2e) and 2-arylethoxyclovan-9-ols (2f-2i) are summarized in Figure 2. Compounds $\mathbf{2 a}-\mathbf{2 g}$ were obtained by the treatment of caryophyllene oxide with a catalytic amount of tetracyanoethylene (TCNE), dissolved in the appropriate alcohol, at room temperature [37]. On the other hand, compounds $\mathbf{2} \mathbf{h}$ and $\mathbf{2} \mathbf{i}$ were obtained in a modified procedure, by the treatment of caryophyllene oxide with a catalytic amount of TCNE and 1.3-1.4 equivalents of the appropriate alcohol, dissolved in propan-2-one, at room temperature. These compounds were identified by their ${ }^{1} \mathrm{H}$ (see Table 2) and ${ }^{13} \mathrm{C}$ (see Table 3) NMR spectra, combined with 1D and 2D NMR techniques and by comparison with authentic samples, where appropriate. All synthesized compounds (2a-2i) displayed the characteristic pattern of ${ }^{1} \mathrm{H}$ and ${ }^{13} \mathrm{C}$ NMR signals of clovan-9-ols, bearing different chains at carbon $\mathrm{C}-2$. The compounds2a [37], 2c [48], 2e [48], and2i [24] were previously described in the literature. A revised assignment of ${ }^{1} \mathrm{H}$ and ${ }^{13} \mathrm{C}$ NMR spectra for compound $2 \mathbf{i}$ together with selected $\mathrm{HMBC}$ correlations can be found in the Supporting Information section.

HREIMS of compounds $\mathbf{2} \mathbf{b}$ and $\mathbf{2 d}$ gave molecular ion peaks at $m / z 266.2268$ and 294.2520, respectively, which were consistent with the molecular formulas $\mathrm{C}_{17} \mathrm{H}_{30} \mathrm{O}_{2}$ and $\mathrm{C}_{19} \mathrm{H}_{34} \mathrm{O}_{2}$. Compound $\mathbf{2 b}$ showed ${ }^{1} \mathrm{H}$ NMR signals at $\delta_{\mathrm{H}} 3.56-3.43(2 \mathrm{H})$ and $1.16(3 \mathrm{H}, \mathrm{t}$, 
$J=7.0 \mathrm{~Hz}$ ), corresponding to an ethoxy chain at C-2. On the other hand, compound $\mathbf{2 d}$ showed ${ }^{1} \mathrm{H}$ NMR signals at $\delta_{\mathrm{H}} 3.44-3.39(2 \mathrm{H}), 1.51(2 \mathrm{H}, \mathrm{m}), 1.36(2 \mathrm{H}, \mathrm{m})$, and $0.89(3 \mathrm{H}$, $\mathrm{t}, J=7.3 \mathrm{~Hz}$ ), assignable to a butoxy chain at C-2. These data led to the assignment of the structures for compounds $\mathbf{2} \mathbf{b}$ and $\mathbf{2} \mathbf{d}$ as $(2 S, 9 R)-2$-ethoxyclovan-9-ol and $(2 S, 9 R)-2$ butoxyclovan-9-ol, respectively.

Compound $2 \mathrm{f}$ showed ${ }^{1} \mathrm{H}$ NMR signals at $\delta_{\mathrm{H}} 3.63-3.53(2 \mathrm{H}), 2.79(2 \mathrm{H}, \mathrm{t}, J=7.2 \mathrm{~Hz})$, and 7.23-7.10 $(5 \mathrm{H})$, which suggested the presence of a phenylethoxy moiety. Its HRESIMS gave a sodium adduct ion peak at $m / z 365.2467[\mathrm{M}+\mathrm{Na}]^{+}$, which is consistent with the formula $\mathrm{C}_{23} \mathrm{H}_{34} \mathrm{O}_{2}+\mathrm{Na}$ for the molecular ion. These data led to the assignment of the structure for compound $\mathbf{2 f}$ as $(2 S, 9 R)-2-\left(2^{\prime}\right.$-phenylethoxy)clovan-9-ol.

The spectroscopy data of compound $\mathbf{2 g}$ were very similar to those of $\mathbf{2 f}$. A comparison of the ${ }^{1} \mathrm{H}$ NMR spectra of both compounds showed as the main difference, the deshielding of the signal corresponding to $\mathrm{H}-2^{\prime}$ from $2.79 \mathrm{ppm}$ in $\mathbf{2 f}$ to $4.03 \mathrm{ppm}$ in $\mathbf{2 g}$ (Table 2). This, together with the presence in the ${ }^{13} \mathrm{C}$ NMR spectrum of two methylenes, one of them bearing an oxygen function, and five aromatic methines, in addition to the fifteen characteristic carbons of the clovane skeleton (Table 3), pointed to the presence of a phenoxyethoxy moiety at C-2. On the other hand, HRESIMS of compound $2 \mathrm{~g}$ gave a sodium adduct ion peak at $m / z 381.2404[\mathrm{M}+\mathrm{Na}]^{+}$, which is consistent with the formula $\mathrm{C}_{23} \mathrm{H}_{34} \mathrm{O}_{3}+\mathrm{Na}$. These data led to the assignment of the structure for compound $2 \mathrm{~g}$ as $(2 S, 9 R)-2-\left(2^{\prime}\right.$-phenoxyethoxy)clovan-9-ol.

Compound $\mathbf{2 h}$ showed a sodium adduct ion at $\mathrm{m} / z 410.2300[\mathrm{M}+\mathrm{Na}]^{+}$, consistent with the formula $\mathrm{C}_{23} \mathrm{H}_{33} \mathrm{NO}_{4}+\mathrm{Na}$, as deduced from the HRESIMS analysis. This, together with ${ }^{1} \mathrm{H}$ NMR signals at $\delta_{\mathrm{H}} 7.38(2 \mathrm{H}, \mathrm{d}, J=8.7 \mathrm{~Hz})$ and $8.12(2 \mathrm{H}, \mathrm{d}, J=8.7 \mathrm{~Hz})$ and infrared absorptions at 1510 and $1344 \mathrm{~cm}^{-1}$, suggested the presence of a nitro-aromatic moiety. These data supported the assignment of the structure of compound $\mathbf{2 h}$ as $(2 S, 9 R)-2-\left(2^{\prime}-(p-\right.$ nitrophenyl)ethoxy)clovan-9-ol.

On the other hand, the treatment of $(1 R, 5 R, 9 S)$-caryophylla-4(12),8(13)-dien-5-ol (3) with TCNE in methanol leads to $(8 R, 9 R)-8$-methoxyisocaryolan-9-ol (4a), together with $(8 R, 9 R)$-isocaryolan-9-one (5) and $(1 S, 2 S, 5 R, 8 S)$-8-methylene-1,4,4-trimethyltricyclo[6 .2.1. $\left.0^{2,5}\right]$ undecane-8-carbaldehyde (6) [36]. Extension of this procedure to higher alcohols led to inseparable mixtures. Alternatively, the treatment of caryophylladienol 3 with a catalytic amount of $\mathrm{Sn}(\mathrm{OTf})_{2}$, dissolved in the appropriate alcohol (from methanol to pentan-1-ol), led to the preparation of a series of $(8 R, 9 R)-8$-alkoxyisocaryolan-9-ols $(\mathbf{4 a}-\mathbf{4} \mathbf{e})$, with yields ranging from $34 \%$ to $42 \%$, together with variable amounts of isocaryolanone 5 and aldehyde 6 (Figure 3). These compounds were identified by their ${ }^{1} \mathrm{H}$ and ${ }^{13} \mathrm{C}$ NMR spectra (see Table 5), combined with 1D and 2D NMR techniques and by comparison with authentic samples, where appropriate. While compound 4 a was previously described by us [36], compound $\mathbf{4 b}$ was reported as rumphellol B, a metabolite of the gorgonian coral Rumphella antipathies [49].

$(8 R, 9 R)$-8-Alkoxyisocaryolan-9-ols $4 \mathbf{c}$ to $4 \mathbf{e}$ displayed the characteristic pattern of ${ }^{1} \mathrm{H}$ and ${ }^{13} \mathrm{C}$ NMR signals of this family of compounds (Table 5). So, compound $4 \mathrm{c}$ showed signals at $\delta_{\mathrm{H}} 3.56(1 \mathrm{H}, \mathrm{dd}, J=10.8,6.3 \mathrm{~Hz})$, corresponding to $\mathrm{H}-9 \beta$, a multiplet between 3.40-3.28 ppm, assigned to the methylene $C-1^{\prime}$ of the lateral chain at $C-8$, and three singlet signals integrating for three protons at $\delta_{\mathrm{H}} 0.97,0.96$, and $0.79 \mathrm{ppm}$, characteristic of the methyl groups $\mathrm{C}-13$ to $\mathrm{C}-15$. In addition, it can be observed there was a triplet signal integrating for three protons at $\delta_{\mathrm{H}} 0.88(J=7.4 \mathrm{~Hz})$, which was assigned to the methyl group of the chain at $\mathrm{C}-8$, and two doublets, at $\delta_{\mathrm{H}} 1.87$ and $0.89(\mathrm{~J}=13.0 \mathrm{~Hz})$, corresponding to each of the protons on carbon $\mathrm{C}-12$. These data led to the assignment of the structure for compound $4 \mathbf{c}$ as $(8 R, 9 R)-8$-propoxyisocaryolan-9-ol. All the carbons and their associated proton signals were assigned using the COSY, HSQC and HMBC spectra.

HREIMS of compounds $4 \mathbf{d}$ and $4 \mathbf{e}$, obtained as colorless oils, indicated the presence of molecular ions at $m / z 294.2592$ and 308.2733, which corresponded with the molecular formulas of $\mathrm{C}_{19} \mathrm{H}_{34} \mathrm{O}_{2}$ and $\mathrm{C}_{20} \mathrm{H}_{36} \mathrm{O}_{2}$, consistent with the structures proposed respectively, for compounds $4 \mathbf{d}$ and $4 \mathrm{e}$. 
The ${ }^{1} \mathrm{H}$ NMR signals of compounds $4 \mathbf{d}$ and $4 \mathbf{e}$ resembled those of the previously described (8R,9R)-8-alkoxyisocaryolan-9-ols $4 \mathbf{a}$ to $4 \mathbf{c}$. On the other hand, their ${ }^{13} \mathrm{C} N M R$ and HSQC spectra showed significant differences, in addition to the characteristic 15 carbons of the isocaryolane skeleton. While compound $\mathbf{4 d}$ presented three methylenes, one of them bearing an oxygen function, and a methyl group, compound $4 \mathbf{e}$ possessed a methyl group and four methylenes, one of them bearing an oxygen function, which is consistent for the presence of a butoxy chain for compound $\mathbf{4 d}$ and a pentoxy chain in compound $4 \mathbf{e}$. These observations, together with a careful analysis of the COSY and HMBC experiments, led to the identification of compound $\mathbf{4} \mathbf{d}$ as $(8 R, 9 R)-8$-butoxyisocaryolan-9-ol and of compound $4 \mathbf{e}$ as $(8 R, 9 R)-8$-pentoxyisocaryolan-9-ol.

The antifungal properties of compounds $2 \mathbf{a}-\mathbf{2} \mathbf{i}$ and $\mathbf{4 a}-\mathbf{4 e}$, at a $10^{-4} \mathrm{M}$ dose, which is a lower dose than the one previously reported for 2-alkoxyclovan-9-ols [38], were determined against the growth of B. cinerea using the poisoned food technique [50]. The commercial fungicide Euparen ${ }^{\circledR}$ (dichlofluanid) was used as a standard for comparison in this test. Several levels of inhibition were observed (Figure 4). For compounds 2a-2e, where the side chain at C-2 varies from methoxy to pentoxy substituents, a tendency of increasing activities was observed, reaching a maximum for (2S,9R)-2-butoxyclovan-9-ol (2d) (Figure 4). These results were consistent with the suggested predictions resulting from a QSAR model published previously for this class of compounds [38]. See a discussion on the correlation between the antifungal activity and $\log P$ below.

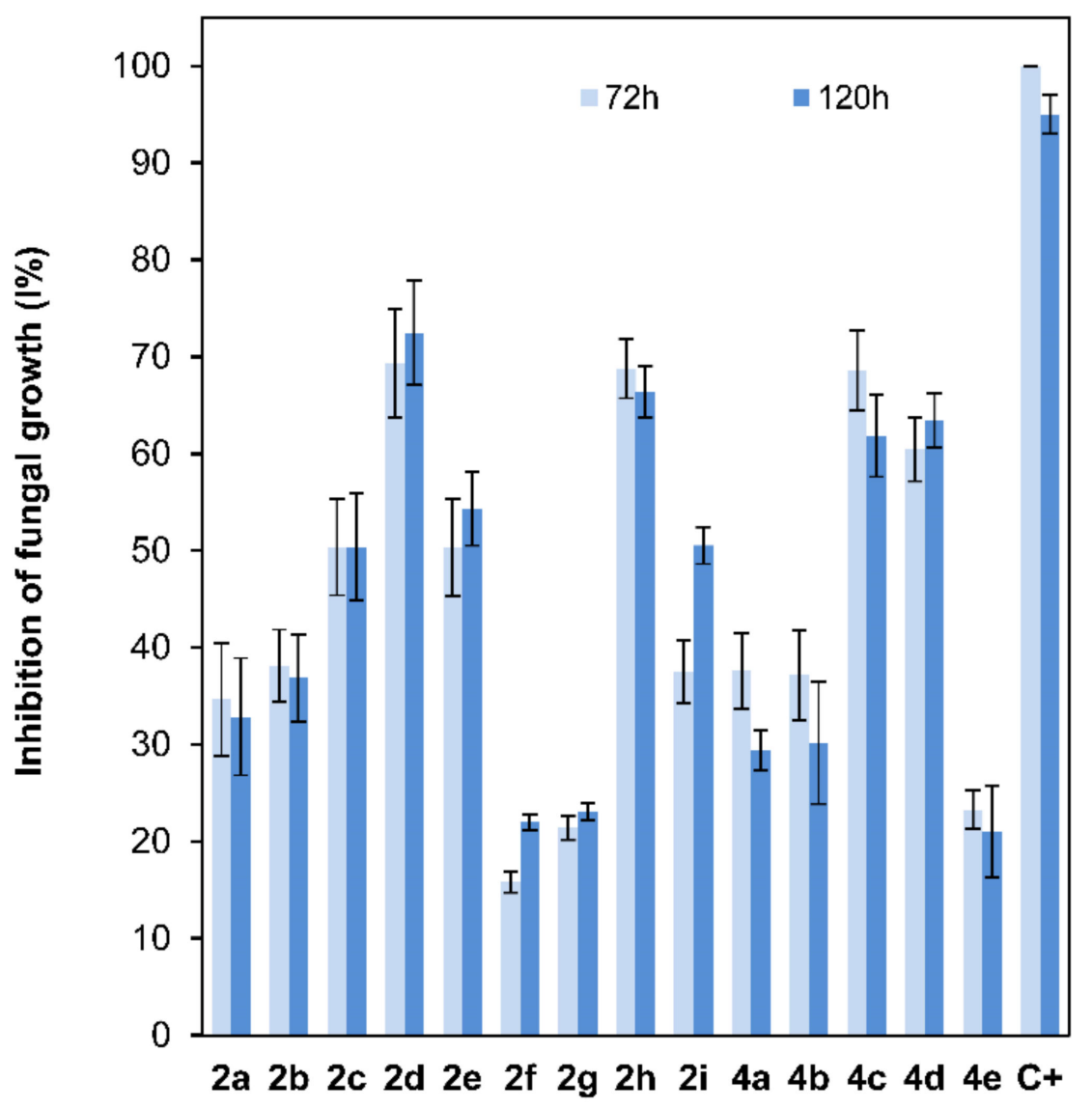

Figure 4. Comparison of the fungal growth inhibition percentage (B. cinerea, 72 and 120h) among compounds 2a-2e (10 ${ }^{-4}$ M dose; 25 ppm 2a, 27 ppm 2b, 28 ppm 2c, 29 ppm 2d, and 31 ppm 2e), 2f-2i (10 ${ }^{-4}$ M dose; 34 ppm 2f, 35 ppm 2g, 38 ppm 2h, and 40 ppm 2i), 4a-4e (10 ${ }^{-4}$ M dose; 25 ppm 4a, 27 ppm 4b, 28 ppm 4c, 29 ppm 4d, and 31 ppm 4e) and dichlofluanid (C+) $\left(10^{-4} \mathrm{M}\right.$ dose; 33 ppm C+). 
On the other hand, the antifungal activities of clovan-9-ol derivatives $O$-substituted at C-2, do not depend only on the lipophilic character of the side chain [38]. For instance, as we have described in our previous work [38], compounds including nitrogen atoms in this side chain at C-2, such as $(2 S, 9 R)-2-\left(2^{\prime}-(p\right.$-nitrophenoxy)ethoxy)clovan-9-ol (2i), display good levels of activity at doses above $50 \mathrm{ppm}$. In order to evaluate the different contributions of the structural components of the aryl substituent on the antifungal activity, compounds $\mathbf{2} \mathbf{f}-\mathbf{2} \mathbf{i}$ were prepared, as described above, and their antifungal properties were compared with those of compounds $\mathbf{2 a - 2 e}$ (Figure 4). Compounds $\mathbf{2} \mathbf{f}$ and $\mathbf{2 g}$, which do not present a $p$-nitro group, displayed antifungal activities lower than those of $(2 S, 9 R)-2$ methoxyclovan-9-ol (2a), the less active compound on the homologous series described above, while $p$-nitro containing compounds showed increased levels of activity. The most active compound of this series, $(2 S, 9 R)-2-\left(2^{\prime}-(p\right.$-nitrophenyl)ethoxy)clovan-9-ol (2h), presented an activity profile comparable to the one of $(2 S, 9 R)$-2-butoxyclovan-9-ol (2d), the most active compound in the above described homologous series (Figure 4), which is an improvement on previously described data [38], where a higher concentration was used (50 ppm). Compounds $\mathbf{2 a}$ and $\mathbf{2} \mathbf{i}$, also evaluated in a previous study [38], are included here for comparison purposes.

To shed some light on the influence of the length of the alkoxy chain at C-8 on the antifungal activity of isocaryolane derivatives, a structural feature which is expected to undergo metabolism by $B$. cinerea $[41,43]$, a parallel study of the antifungal activities of compounds $\mathbf{4 a}-\mathbf{4 e}$ on the growth of $B$. cinerea was carried out. Compounds $(8 R, 9 R)-8$ propoxyisocaryolan-9-ol (4c) and (8R,9R)-8-butoxyisocaryolan-9-ol (4d) were the most active compounds in this series. The activity profiles observed for both compounds were similar to the ones shown for $(2 S, 9 R)-2$-butoxyclovan-9-ol (2d) and $(2 S, 9 R)-2-\left(2^{\prime}-(p-\right.$ nitrophenyl)ethoxy)clovan-9-ol (2h) (Figure 4).

Most of the active compounds in this study (2d, 2h, $4 \mathbf{c}$ and $4 \mathrm{~d})$ present a calculated [51] $\log p$ near to the upper limit for this parameter, established by the Lipinsky "rule of five" for the oral availability of drugs-most molecules which show activity via oral administration have $\log p<5$, molecular weight $<500$ and should contain no more than 10 hydrogen bond acceptors and less than 5 hydrogen bond donors-[52] (compound 2d, $\log p=4.499 ;$ compound $2 \mathrm{~h}, \log p=4.824$; compound $4 \mathrm{c}, \log p=4.310$ and compound $\mathbf{4 d}, \log p=4.870)$. Compounds with longer lipophilic aliphatic chains like $\mathbf{2 e}$ and $4 \mathbf{e}$ show a decreased activity, presenting $\log p$ values above five (compound $2 \mathbf{e}, \log p=5.004$; compound $\mathbf{4 e}, \log p=5.370$ ). For 2 -alkyloxyclovan-9-ols $\mathbf{2 a - 2} \mathbf{c}$, a roughly linear correlation between $\log p$ values and activity (Figure 4) can be established (compound 2a, $\log p=3.061$; compound $\mathbf{2 b}, \log p=3.437$; compound $\mathbf{2} \mathbf{c}, \log p=3.939)$. This is not the case for either 2-arylalkyloxyclovan-9-ols (2f-2i) or 8-alkyloxyisocaryolan-9-ols (4a-4d), where additional factors, like electronic distribution, must be taken in account.

The effects of previously reported $(2 S, 9 R)-2-$ metoxyclovan-9-ol (2a) [23], $(2 S, 9 R)$ 2-propoxyclovan-9-ol (2c) [48], (2S,9R)-2-pentoxyclovan-9-ol (2e) [48], (2S,9R)-2-(2'-( $p$ nitrophenoxy)ethoxy)clovan-9-ol (2i) [38], (8R,9R)-8-methoxyisocaryolan-9-ol (4a) [36] and $(8 R, 9 R)-8$-ethoxyisocaryolan-9-ol (4b) [32], as well as the clovane derivatives $\mathbf{2} \mathbf{b}, \mathbf{2} \mathbf{d}$, and $\mathbf{2 f}-\mathbf{2 h}$, and isocaryolane derivatives $\mathbf{4 c}-\mathbf{4 e}$, prepared as described above, were evaluated on the germination (Figure 5) and radicle and shoot growth (Figure 6) of L. sativa (lettuce). L. sativa seeds are widely used to evaluate phytotoxicity [53-55] because of their ready availability and their suitable germination characteristics [45]. Concentrations of $10^{-3} \mathrm{M}$ and $10^{-5} \mathrm{M}$ were evaluated, as they encompassed the doses used for the evaluation of the inhibition of growth of $B$. cinerea $\left(10^{-4} \mathrm{M}\right)$. 


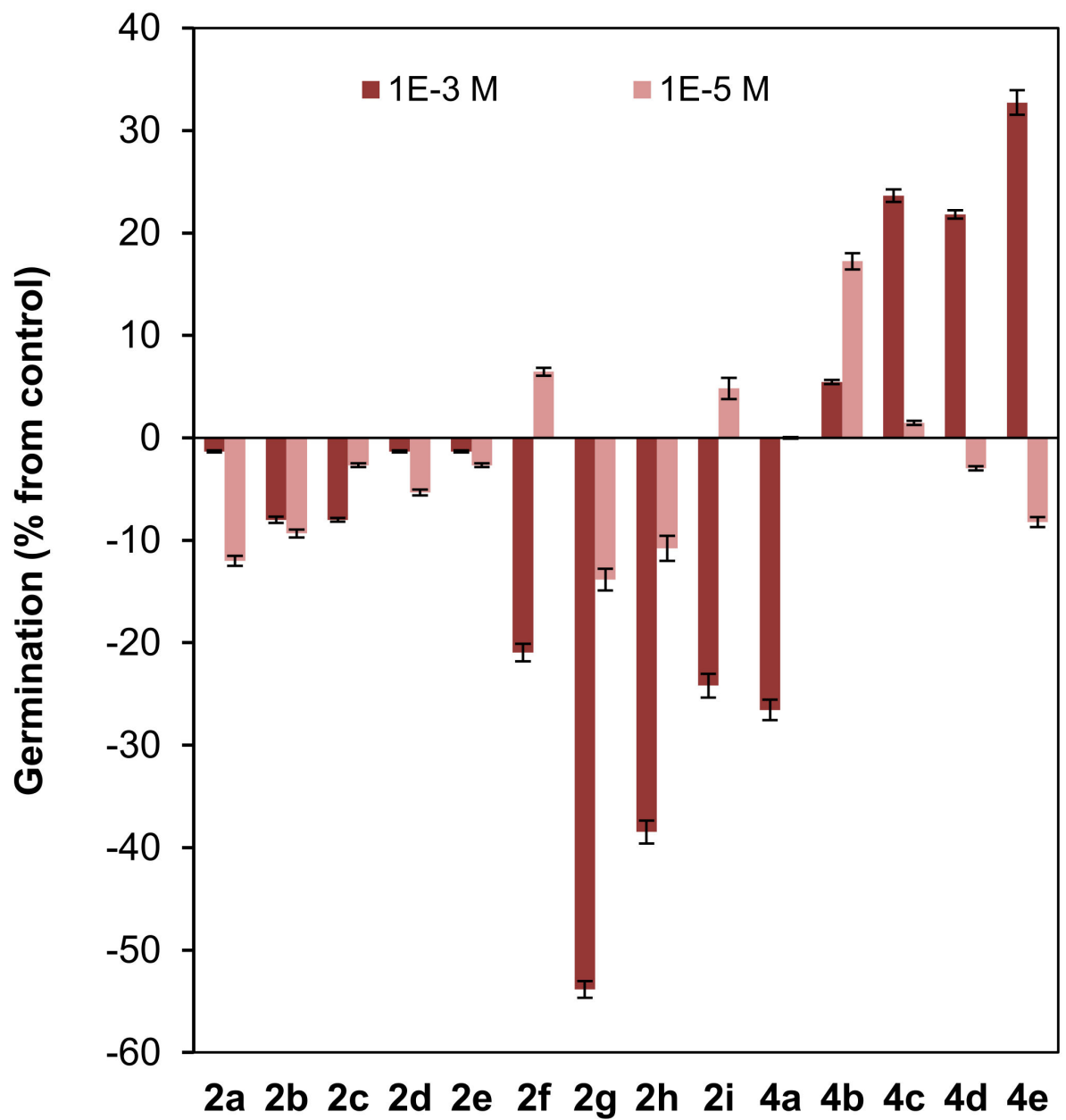

Figure 5. Effect of clovane derivatives $\mathbf{2 a}-\mathbf{2} \mathbf{i}$ and isocaryolane derivatives $\mathbf{4 a}-\mathbf{4} \mathbf{e}$ on the germination of L. sativa (see Figures 2 and 3). Values are presented as percentage differences from the control, where a positive value represents stimulation of growth and a negative value represents inhibition.

A low inhibitory effect on germination was observed for the homologous series of clovane derivatives $\mathbf{2 a - 2 e}$, with a maximum effect found for ethoxy and propoxy derivatives (2b and $2 \mathbf{c}$ ) (Figure 5).

On the other hand, the introduction of an aromatic moiety in the side chain of clovane derivatives (compounds $\mathbf{2} \mathbf{f}-\mathbf{2} \mathbf{i}$ ), sharply increased the inhibitory effect on germination at the highest concentration used $\left(10^{-3} \mathrm{M}\right)$. Furthermore, this effect is exacerbated when either a nitro or an alkoxy moiety is connected to the aromatic ring (see compounds $\mathbf{2} \mathbf{g}$ and $\mathbf{2 h}$ ). Noteworthily, the simultaneous presence of both moieties, arranged in a 1,4 substitution pattern, led to a cancellation of the above mentioned effects, as germination inhibition by compound $2 \mathrm{i}$ is similar to that shown by $(2 S, 9 R)-2-\left(2^{\prime}-\right.$ phenylethoxy)clovan-9-ol (2f).

Interestingly, and referring also to the highest concentration evaluated $\left(10^{-3} \mathrm{M}\right)$, while $(8 R, 9 R)-8-m e t h o x y i s o c a r y o l a n-9-o l$ (4a) showed a germination inhibitory effect comparable to that of the clovane derivatives $\mathbf{2} \mathbf{f}$ and $\mathbf{2} \mathbf{i}$, an opposite effect was observed for the homologous series of isocaryolane derivatives $\mathbf{4 b}-\mathbf{4 e}$, where the maximum stimulatory effect was observed for $(8 R, 9 R)-8$-pentoxyisocaryolan-9-ol (4e).

Different patterns were shown for the effect of clovane derivatives on radicle and shoot growth (Figure 6). On one hand, alkoxyclovanols $\mathbf{2 a - 2 e}$ showed a general inhibitory effect on shoot growth at the highest concentration evaluated $\left(10^{-3} \mathrm{M}\right)$, with a maximum inhibitory effect observed for (2S,9R)-2-propoxyclovan-9-ol (2c). A different response was found for this homologous series $(\mathbf{2 a}-\mathbf{2 e})$ on radicle growth, where a progression on the length of the alkyl chain leads to activity changes from inhibitory to stimulatory. A maximum stimulatory effect was found for $(2 S, 9 R)$-2-butoxyclovan-9-ol (2d), the most 
effective alkoxyclovanol of the homologous series $\mathbf{2 a}-\mathbf{2 e}$, for the inhibition of the growth of B. cinerea $\left(10^{-4} \mathrm{M}\right.$ dose, see Figure 4$)$. On the other hand, while there was almost no effect of $(2 S, 9 R)-2-\left(2^{\prime}\right.$-phenoxyethoxy)clovan-9-ol (2g) on shoot growth, and a moderate inhibitory effect on radicle growth, there was a general stimulatory effect, on both shoot and radicle growth, for compounds $\mathbf{2} \mathbf{f}, \mathbf{2} \mathbf{h}$, and $\mathbf{2} \mathbf{i}$, where $\mathbf{2} \mathbf{i}$ showed the greatest stimulatory effect. Consequently, given that (2S,9R)-2-(2'-( $p$-nitrophenyl)ethoxy)clovan-9-ol (2h) showed an inhibitory effect on the growth of $B$. cinerea of a similar magnitude to that of the most effective alkoxyclovanol of the homologous series (2d) $\left(10^{-4} \mathrm{M}\right.$ dose, see Figure 4$)$, this made compound $2 \mathrm{~h}$ the most potentially useful of the clovane derivatives evaluated so far, as the inhibition of germination was compensated with stimulation of both radicle and shoot growth. Compound $\mathbf{2 d}$, with a comparable inhibitory effect on B. cinerea growth (see Figure 4), showed inhibitory effects on both germination and shoot growth and only a moderate stimulatory effect on radicle growth (see Figure 6).
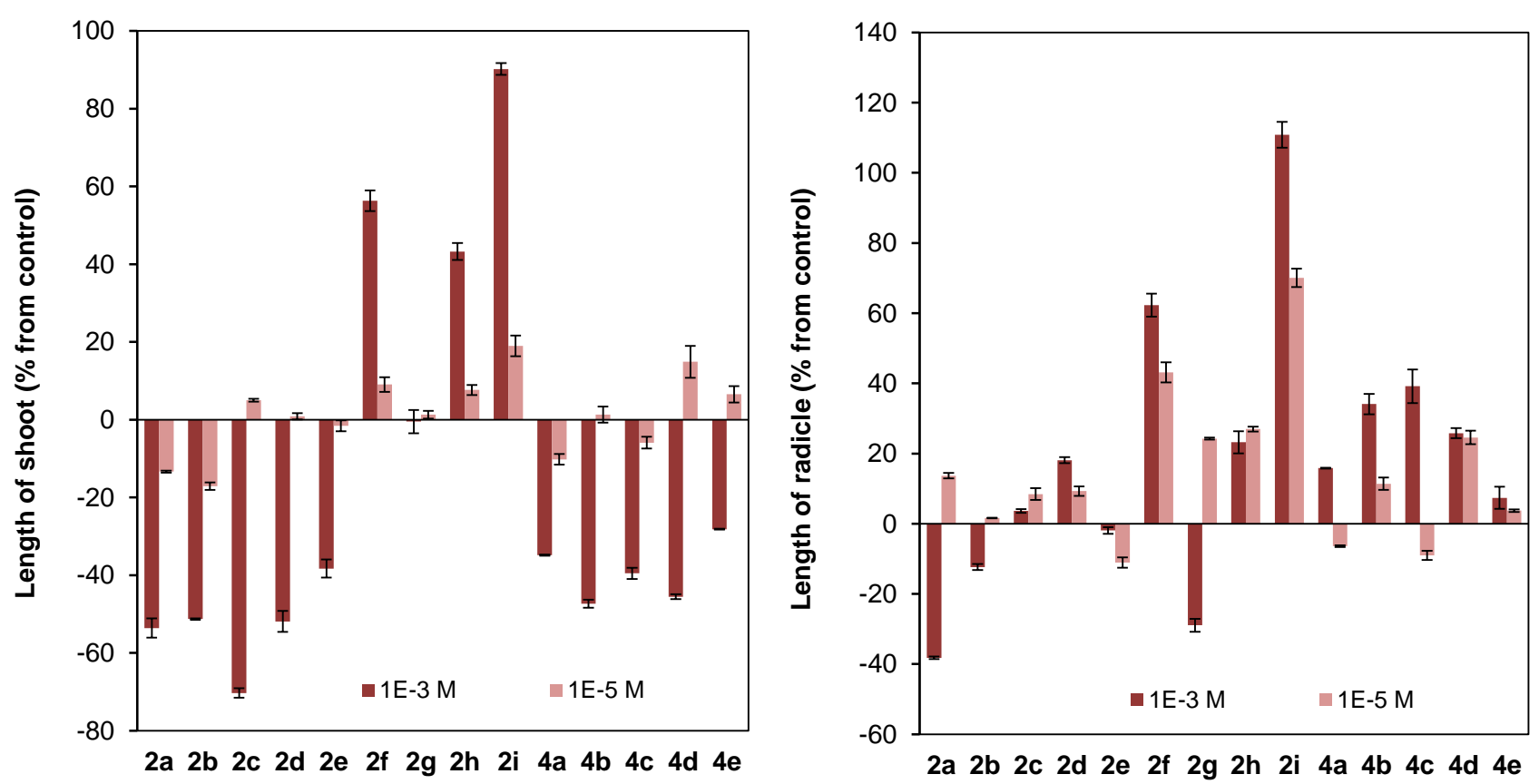

Figure 6. Effect of clovane derivatives $\mathbf{2 a}-\mathbf{2} \mathbf{i}$ and isocaryolane derivatives $\mathbf{4 a}-\mathbf{4 e}$ on radicle and shoot growth of $L$. sativa (see Figures 2 and 3). Values are presented as percentage differences from the control, where a positive value represents stimulation of growth and a negative value represents inhibition.

On the other hand, different trends could be found for the isocaryolane derivatives evaluated. Along the homologous series $4 \mathbf{a}-\mathbf{4} \mathbf{e}$, an increase of the stimulation of germination could be found at the highest dose evaluated $\left(10^{-3} \mathrm{M}\right)$, as discussed above (Figure 5). Additionally, also at the $10^{-3} \mathrm{M}$ dose, different tendencies could be found for radicle and shoot growth (Figure 6); while a general stimulatory effect was apparent for radicle growth, with a maximum effect found for $(8 R, 9 R)-8$-propoxyisocaryolan-9-ol $(4 \mathbf{c})$, a general inhibitory effect on shoot growth was observed. Given that ( $8 R, 9 R)-8$-propoxyisocaryolan9-ol (4c) had inhibitory activity on the growth of $B$. cinerea of a similar magnitude to the most effective alkoxyclovanol, compound $\mathbf{2 d}$ (see Figure 4), this made compound $\mathbf{4} \mathbf{c}$ the most potentially useful of the isocaryolane derivatives evaluated so far.

\section{Conclusions}

In conclusion, a high activity against $B$. cinerea can be found in 2-alkoxyclovan-9-ols (2a-2e), 2-arylalkoxyclovan-9-ols (2f-2i) or 8-alkoxyisocaryolan-9-ols (4a-4d) at a $10^{-4} \mathrm{M}$ dose, depending on the nature of the substituent side chain (see Figure 4), where compounds $\mathbf{2 d}, \mathbf{2 h}, \mathbf{4 c}$ and $\mathbf{4 d}$ show the best activities, even improving on those described before [38]. 
On the other hand, in the dose range $10^{-3} \mathrm{M}$ to $10^{-5} \mathrm{M}$, different phytotoxicity profiles can be associated to the structural classes mentioned above. In general terms, 2-alkoxyclovan-9-ols (2a-2e) exert a phytotoxic effect, especially on shoot growth (see Figure 6). 2-Arylalkoxyclovan-9-ols (2f-2i) are phytotoxic on germination (see Figure 5), but stimulate shoot and radicle growth (Figure 6), which would make them suitable candidates for post-germination applications, with compound $2 \mathrm{~h}$ being the best candidate. Finally, 8-alkoxyisocaryolan-9-ols (4a-4d) inhibit shoot growth (Figure 6), but stimulate germination (Figure 5) and radicle growth (Figure 6), which would make them suitable candidates for both pre- and post-germination applications, with compounds $4 \mathbf{c}$ and $4 \mathrm{~d}$ being the best candidates.

Supplementary Materials: The following are available online at https:/ /www.mdpi.com/article/10 $.3390 /$ jof7121079/s1, Revised assignments of ${ }^{1} \mathrm{H}$ and ${ }^{13} \mathrm{C}$ NMR spectra for compound $2 \mathbf{i}$, together with selected HMBC correlations. Table S1. Figures S1-S20a. ${ }^{1} \mathrm{H}$ - and ${ }^{13} \mathrm{C}$ NMR spectra of compounds $2 b, 2 d, 2 f-2 i$, and $4 b-4 e$.

Author Contributions: Conceptualisation, I.G.C., R.D.-P. and A.J.M.-S.; methodology, A.d.A.P.B., J.A., G.G.d.S. and T.S.O.; validation, M.A.D.B., J.A.T., I.G.C., R.D.-P. and A.J.M.-S.; investigation, A.R.-G., A.A.P., M.A.D.B. and J.A.T.; data curation, A.d.A.P.B., J.A., G.G.d.S., T.S.O., A.R.-G. and A.A.P.; writing—original draft preparation, A.J.M.-S., R.D.-P., I.G.C., M.A.D.B. and J.A.T; writingreview and editing, I.G.C., R.D.-P., A.J.M.-S. and J.A.T.; supervision, A.J.M.-S. and I.G.C.; project administration, I.G.C.; funding acquisition, I.G.C., A.J.M.-S. and R.D.-P.; contributed equally to this work A.d.A.P.B. and J.A. All authors have read and agreed to the published version of the manuscript.

Funding: This research was supported by a grant from Ministerio de Ciencia, Innovación y Universidades (RTI2018-097356-B-C21, MCIU/AEI/FEDER, EU). A.d.A.P.B., G.G.d.S. and J.A.T. thank Coordenação de Aperfeiçoamento de Pessoal de nível superior (CAPES, Brazil) for research fellowships (CAPES-DGU 197/09).

Institutional Review Board Statement: Not applicable.

Informed Consent Statement: Not applicable.

Data Availability Statement: Not applicable.

Acknowledgments: Dedicated to James R. Hanson (University of Sussex, UK). The use of NMR and MS facilities at the Servicios Centrales de Investigación Científica y Tecnológica (SC-ICYT) of the University of Cádiz is acknowledged.

Conflicts of Interest: The authors declare no conflict of interest.

\section{References}

1. Elad, Y.; Pertot, I.; Cotes Prado, A.M.; Stewart, A. Plant Hosts of Botrytis Spp. In Botrytis-The Fungus, the Pathogen and Its Management in Agricultural Systems; Fillinger, S., Elad, Y., Eds.; Springer International Publishing: Dordrecht, The Netherlands, 2016; pp. 413-486.

2. Carisse, O. Epidemiology and Aerobiology of Botrytis Spp. In Botrytis-The Fungus, the Pathogen and Its Management in Agricultural Systems; Fillinger, S., Elad, Y., Eds.; Springer International Publishing: Dordrecht, The Netherlands, 2016; pp. 127-148.

3. McGrath, M.T. What Are Fungicides? Available online: http://www.apsnet.org/edcenter/intropp/topics/Pages/Fungicides. aspx (accessed on 11 November 2021).

4. Fenner, K.; Canonica, S.; Wackett, L.P.; Elsner, M. Evaluating Pesticide Degradation in the Environment: Blind Spots and Emerging Opportunities. Science 2013, 341, 752-758. [CrossRef] [PubMed]

5. Smith, A.M.; Gilbertson, L.M. Rational Ligand Design To Improve Agrochemical Delivery Efficiency and Advance Agriculture Sustainability. ACS Sustain. Chem. Eng. 2018, 6, 13599-13610. [CrossRef]

6. Fungicide Resistance Action Committee. Available online: https://www.frac.info/home (accessed on 14 November 2021).

7. Brzozowski, L.; Mazourek, M.A. A Sustainable Agricultural Future Relies on the Transition to Organic Agroecological Pest Management. Sustainability 2018, 10, 2023. [CrossRef]

8. Stenberg, J.A. A Conceptual Framework for Integrated Pest Management. Trends Plant Sci. 2017, 22, 759-769. [CrossRef]

9. Nicot, P.C.; Bardin, M.; Alabouvette, C.; Köhl, J.; Ruocco, M. Potential of Biological Control Based on Published Research. Protection against Plant Pathogens of Selected Crops. In Classical and Augmentative Biological Control Against Diseases and Pests: Critical Status Analysis and Review of Factors Influencing Their Success; IOBC/WPRS, 2011; pp. 1-11. Available online: https:/ /hal.inrae.fr/hal-02809578/ document (accessed on 14 November 2021). 
10. Kim, B.; Han, J.W.; Thi, N.M.; Kim, H.; Choi, G.J.; Kim, B.; Kim, J.-C.; Thi, N.M.; Kim, H.; Choi, G.J.; et al. Identification of novel compounds, oleanane- and ursane-type triterpene glycosides, from Trevesia palmata: Their biocontrol activity against phytopathogenic fungi. Sci. Rep. 2018, 8, 14522-14533. [CrossRef]

11. Teshima, Y.; Ikeda, T.; Imada, K.; Sasaki, K.; El-Sayed, M.A.; Shigyo, M.; Tanaka, S.; Ito, S. Identification and Biological Activity of Antifungal Saponins from Shallot (Allium cepa L. Aggregatum Group). J. Agric. Food Chem. 2013, 61, 7440-7445. [CrossRef]

12. Munafo, J.P.; Gianfagna, T.J. Antifungal activity and fungal metabolism of steroidal glycosides of Easter Lily (Lilium longiflorum Thunb.) by the plant pathogenic fungus, Botrytis cinerea. J. Agric. Food Chem. 2011, 59, 5945-5954. [CrossRef]

13. Fatema, U.; Jensen, D.F.; Karlsson, M.; Dubey, M.; Fatema, U.; Broberg, A. Functional analysis of polyketide synthase genes in the biocontrol fungus Clonostachys rosea. Sci. Rep. 2018, 8, 15009-15026. [CrossRef]

14. Tanaka, K.; Ishihara, A.; Nakajima, H. Isolation of anteiso-C17, iso-C17, iso-C16, and iso-C15 Bacillomycin D from Bacillus amyloliquefaciens SD-32 and Their Antifungal Activities against Plant Pathogens. J. Agric. Food Chem. 2014, 62, 1469-1476. [CrossRef]

15. Liermann, J.C.; Kolshorn, H.; Opatz, T.; Thines, E.; Anke, H. Xanthepinone, an Antimicrobial Polyketide from a Soil Fungus Closely Related to Phoma medicaginis. J. Nat. Prod. 2009, 72, 1905-1907. [CrossRef]

16. Altomare, C.; Perrone, G.; Zonno, M.C.; Evidente, A.; Pengue, R.; Fanti, F.; Polonelli, L. Biological characterization of fusapyrone and deoxyfusapyrone, two bioactive secondary metabolites of Fusarium semitectum. J. Nat. Prod. 2000, 63, 1131-1135. [CrossRef]

17. Xu, D.; Zhang, B.-Y.; Yang, X.-L. Antifungal Monoterpene Derivatives from the Plant Endophytic Fungus Pestalotiopsis foedan. Chem. Biodivers. 2016, 13, 1422-1425. [CrossRef]

18. Hussain, H.; Krohn, K.; Ahmed, I.; Draeger, S.; Schulz, B.; Di Pietro, S.; Pescitelli, G. Phomopsinones A-D: Four New Pyrenocines from Endophytic Fungus Phomopsis sp. Eur. J. Org. Chem. 2012, 1783-1789. [CrossRef]

19. Li, X.-J.; Zhang, Q.; Zhang, A.-L.; Gao, J.-M. Metabolites from Aspergillus fumigatus, an Endophytic Fungus Associated with Melia azedarach, and Their Antifungal, Antifeedant, and Toxic Activities. J. Agric. Food Chem. 2012, 60, 3424-3431. [CrossRef]

20. Narayan, O.P.; Verma, N.; Singh, A.K.; Oelmuller, R.; Kumar, M.; Prasad, D.; Kapoor, R.; Dua, M.; Johri, A.K. Antioxidant enzymes in chickpea colonized by Piriformospora indica participate in defense against the pathogen Botrytis cinerea. Sci. Rep. 2017, 7, 13553. [CrossRef]

21. Bolivar-Anillo, H.J.; Garrido, C.; Collado, I.G. Endophytic microorganisms for biocontrol of the phytopathogenic fungus Botrytis cinerea. Phytochem. Rev. 2020, 19, 721-740. [CrossRef]

22. Dias, M.C. Phytotoxicity: An overview of the physiological responses of plants exposed to fungicides. J. Bot. 2012, $2012,135479$. [CrossRef]

23. Saladin, G.; Magné, C.; Clément, C. Effects of fludioxonil and pyrimethanil, two fungicides used against Botrytis cinerea, on carbohydrate physiology in Vitis vinifera L. Pest Manag. Sci. 2003, 59, 1083-1092. [CrossRef]

24. Petit, A.-N.; Fontaine, F.; Clément, C.; Vaillant-Gaveau, N. Photosynthesis Limitations of Grapevine after Treatment with the Fungicide Fludioxonil. J. Agric. Food Chem. 2008, 56, 6761-6767. [CrossRef]

25. Dias, M.C.; Figueiredo, P.; Duarte, I.F.; Gil, A.M.; Santos, C. Different responses of young and expanded lettuce leaves to fungicide Mancozeb: Chlorophyll fluorescence, lipid peroxidation, pigments and proline content. Photosynthetica 2014, 52, 148-151. [CrossRef]

26. Collado, I.G.; Sánchez, A.J.M.; Hanson, J.R. Fungal terpene metabolites: Biosynthetic relationships and the control of the phytopathogenic fungus Botrytis cinerea. Nat. Prod. Rep. 2007, 24, 674-686. [CrossRef]

27. Collado, I.G.; Hanson, J.R.; Sánchez, A.J.M. The botryane sesquiterpenoid metabolism of the fungus Botrytis cinerea. J. Chem. Res. 2017, 41, 435-440. [CrossRef]

28. Bradshaw, A.P.W.; Hanson, J.R.; Nyfeler, R.; Sadler, I.H. Studies in terpenoid biosynthesis. Part 25. The fate of the mevalonoid hydrogen atoms in the biosynthesis of the sesquiterpenoid, dihydrobotrydial. J. Chem. Soc. Perkin Trans. 1 1982, $2187-2192$. [CrossRef]

29. Bradshaw, A.P.W.; Hanson, J.R.; Nyfeler, R. Studies in terpenoid biosynthesis. Part 24. The formation of the carbon skeleton of the sesquiterpenoid, dihydrobotrydial. J. Chem. Soc. Perkin Trans. 1981, 1, 1469-1472. [CrossRef]

30. Hanson, J.R. The biosynthesis of some sesquiterpenoids. Pure Appl. Chem. 1981, 53, 1155-1162. [CrossRef]

31. Pinedo, C.; Wang, C.M.; Pradier, J.M.; Dalmais, B.; Choquer, M.; Le Pêcheur, P.; Morgant, G.; Collado, I.G.; Cane, D.E.; Viaud, M. Sesquiterpene Synthase from the Botrydial Biosynthetic Gene Cluster of the Phytopathogen Botrytis cinerea. ACS Chem. Biol. 2008, 3, 791-801. [CrossRef]

32. Moraga, J.; Dalmais, B.; Izquierdo-Bueno, I.; Aleu, J.; Hanson, J.R.; Hernández-Galán, R.; Viaud, M.; Collado, I.G. Genetic and molecular basis of botrydial biosynthesis: Connecting cytochrome P450-encoding genes to biosynthetic intermediates. ACS Chem. Biol. 2016, 11, 2838-2846. [CrossRef] [PubMed]

33. Rossi, F.R.; Garriz, A.; Marina, M.; Romero, F.M.; González, M.E.; Collado, I.G.; Pieckenstain, F.L. The sesquiterpene botrydial produced by Botrytis cinerea induces the hypersensitive response on plant tissues and its action is modulated by salicylic acid and jasmonic acid signaling. Mol. Plant-Microbe Interact. 2011, 24, 888-896. [CrossRef]

34. Siewers, V.; Viaud, M.; Jiménez-Teja, D.; Collado, I.G.; Schulze Gronover, C.; Pradier, J.M.; Tudzynsk, B.; Tudzynski, P. Functional analysis of the cytochrome P450 monooxygenase gene bcbot1 of Botrytis cinerea indicates that botrydial is a strain-specific virulence factor. Mol. Plant-Microbe Interact. 2005, 18, 602-612. [CrossRef] 
35. Collado, I.G.; Hanson, J.R.; Macías-Sánchez, A.J. Recent advances in the chemistry of caryophyllene. Nat. Prod. Rep. 1998, 15, 187-204. [CrossRef]

36. Racero, J.C.; Macías-Sánchez, A.J.; Hernández-Galán, R.; Hitchcock, P.B.; Hanson, J.R.; Collado, I.G. Novel Rearrangement of an Isocaryolane Sesquiterpenoid under Mitsunobu Conditions. J. Org. Chem. 2000, 65, 7786-7791. [CrossRef]

37. Collado, I.G.; Hanson, J.R.; Macías-Sánchez, A.J. The cleavage of caryophyllene oxide catalyzed by tetracyanoethylene. Tetrahedron 1996, 52, 7961-7972. [CrossRef]

38. Saiz-Urra, L.; Racero, J.C.; Macías-Sánchez, A.J.; Hernández-Galán, R.; Hanson, J.R.; Pérez- González, M.; Collado, I.G. Synthesis and Quantitative Structure-Antifungal Activity Relationships of Clovane Derivatives against Botrytis cinerea. J. Agric. Food Chem. 2009, 57, 2420-2428. [CrossRef]

39. Collado, I.G.; Hanson, J.R.; Macías-Sánchez, A.J.; Mobbs, D. The Biotransformation of Some Clovanes by Botrytis cinerea. J. Nat. Prod. 1998, 61, 1348-1351. [CrossRef]

40. Deligeorgopoulou, A.; Macías-Sánchez, A.J.; Mobbs, D.J.; Hitchcock, P.B.; Hanson, J.R.; Collado, I.G. Structure-Activity Relationships in the Fungistatic Activity against Botrytis cinerea of Clovanes Modified on Ring C. J. Nat. Prod. 2004, 67, 793-798. [CrossRef]

41. Ascari, J.; Boaventura, M.A.D.; Takahashi, J.A.; Durán-Patrón, R.; Hernández-Galán, R.; Macías-Sánchez, A.J.; Collado, I.G. Biotransformation of bioactive isocaryolanes by Botrytis cinerea. J. Nat. Prod. 2011, 74, 1707-1712. [CrossRef]

42. Durán-Patrón, R.; Cantoral, J.M.; Hernández-Galán, R.; Hanson, J.R.; Collado, I.G. The biodegradation of the phytotoxic metabolite botrydial by its parent organism, Botrytis cinerea. J. Chem. Res. 2004, 6, 441-443. [CrossRef]

43. Ascari, J.; Boaventura, M.A.D.; Takahashi, J.A.; Durán-Patrón, R.; Hernández-Galán, R.; Macías-Sánchez, A.J.; Collado, I.G. Phytotoxic activity and metabolism of Botrytis cinerea and structure-activity relationships of isocaryolane derivatives. J. Nat. Prod. 2013, 76, 1016-1024. [CrossRef]

44. Macías, F.A.; Simonet, A.M.; Esteban, M.D. Potential allelopathic lupane triterpenes from bioactive fractions of Melilotus messanensis. Phytochemistry 1994, 36, 1369-1379. [CrossRef]

45. Macías, F.A.; Castellano, D. Search for a Standard Phytotoxic Bioassay for Allelochemicals. Selection of Standard Target Species. J. Agric. Food Chem. 2000, 48, 2512-2521. [CrossRef]

46. Boaventura, M.A.D.; Pereira, R.G.; Freitas, L.B.O.; Reis, L.A.; Vieira, H.S. Preparation and phytotoxicity of novel kaurane diterpene amides with potential use as herbicides. J. Agric. Food Chem. 2008, 56, 2985-2988. [CrossRef] [PubMed]

47. Rocha, A.D.; dos Santos, G.C.; Fernandes, N.G.; Pfenning, L.H.; Takahashi, J.A.; Boaventura, M.A.D. Hydroxylation at carbon-2 of ent-16-oxo-17-norkauran-19-oic acid by Fusarium proliferatum. J. Nat. Prod. 2010, 73, 1431-1433. [CrossRef] [PubMed]

48. De Souza, G.G.; Oliveira, T.S.; Takahashi, J.A.; Collado, I.G.; Macías-Sánchez, A.J.; Hernández-Galán, R. Biotransformation of clovane derivatives. Whole cell fungi mediated domino synthesis of rumphellclovane A. Org. Biomol. Chem. 2012, 10, 3315-3319. [CrossRef] [PubMed]

49. Chung, H.-M.; Wang, W.-H.; Hwang, T.-L.; Chen, J.-J.; Fang, L.-S.; Wen, Z.-H.; Wang, Y.-B.; Wu, Y.-C.; Sung, P.-J. Rumphellols A and B, new caryophyllene sesquiterpenoids from a Formosan gorgonian coral, Rumphella antipathies. Int. J. Mol. Sci. 2014, 15, 15679-15688. [CrossRef]

50. Patil, I.S.; Kulkarni, S.; Hedge, R.K. Bioassay of fungicides against Drechslera sorokiniana (Sacc). Pesticides 1986, $20,30-31$.

51. Molinspiration Cheminformatics. Available online: http://www.molinspiration.com/ (accessed on 14 November 2021).

52. Lipinski, C.A.; Lombardo, F.; Dominy, B.W.; Feeney, P.J. Experimental and computational approaches to estimate solubility and permeability in drug discovery and development settings. Adv. Drug Deliv. Rev. 2001, 46, 3-26. [CrossRef]

53. Chinchilla, N.; Santana, A.; Varela, R.M.; Fronczek, F.R.; Molinillo, J.M.G.; Macías, F.A. Preparation and Phytotoxicity Evaluation of 11,13-Dehydro seco-Guaianolides. J. Nat. Prod. 2019, 82, 2501-2508. [CrossRef]

54. Ríos, M.Y.; Córdova-Albores, L.C.; Ramírez-Cisneros, M.Á.; King-Díaz, B.; Lotina-Hennsen, B.; León Rivera, I.; Miranda-Sánchez, D. Phytotoxic Potential of Zanthoxylum affine and Its Major Compound Linarin as a Possible Natural Herbicide. ACS Omega 2018, 3, 14779-14787. [CrossRef]

55. Ren, X.; He, X.; Zhang, Z.; Yan, Z.; Jin, H.; Li, X.; Qin, B. Isolation, Identification, and Autotoxicity Effect of Allelochemicals from Rhizosphere Soils of Flue-Cured Tobacco. J. Agric. Food Chem. 2015, 63, 8975-8980. [CrossRef] 